\title{
KRAS-dependent sorting of miRNA to exosomes
}

\author{
Diana J Cha ${ }^{1,2 \dagger}$, Jeffrey L Franklin ${ }^{3,4,5+}$, Yongchao Dou ${ }^{6}, \mathrm{Qi}_{\mathrm{Liu}}{ }^{6}$, \\ James N Higginbotham ${ }^{3,4}$, Michelle Demory Beckler ${ }^{4}$, Alissa M Weaver ${ }^{3,7,8}$, \\ Kasey Vickers ${ }^{9}$, Nirpesh Prasad ${ }^{10}$, Shawn Levy ${ }^{10}$, Bing Zhang ${ }^{6}$, \\ Robert J Coffey ${ }^{3,4,5 *}$, James G Patton ${ }^{1,2 *}$
}

1Department of Biological Sciences, Vanderbilt University Medical Center, Nashville, United States; ${ }^{2}$ Vanderbilt University, Nashville, United States; ${ }^{3}$ Department of Cell and Developmental Biology, Vanderbilt University Medical Center, Nashville, United States; ${ }^{4}$ Department of Medicine, Vanderbilt University Medical Center, Nashville, United States; ${ }^{5}$ Affairs Medical Center, Nashville, United States; ${ }^{6}$ Department of Biomedical Informatics, Vanderbilt University Medical Center, Nashville, United States; ${ }^{7}$ Department of Cancer Biology, Vanderbilt University Medical Center, Nashville, United States; ${ }^{8}$ Department of Pathology, Microbiology and Immunology, Vanderbilt University Medical Center, Nashville, United States; ${ }^{9}$ Department of Cardiology, Vanderbilt University Medical Center, Nashville, United States; ${ }^{10}$ HudsonAlpha Institute for Biotechnology, Huntsville, United States
*For correspondence: robert. coffey@vanderbilt.edu (RJC); james.g.patton@vanderbilt. edu (JGP)

†These authors contributed equally to this work

Competing interests: The authors declare that no competing interests exist.

Funding: See page 19

Received: 26 February 2015

Accepted: 29 June 2015

Published: 01 July 2015

Reviewing editor: Phillip D Zamore, Howard Hughes Medical Institute, University of Massachusetts Medical School, United States

Copyright Cha et al. This article is distributed under the terms of the Creative Commons Attribution License, which permits unrestricted use and redistribution provided that the original author and source are credited.
Abstract Mutant KRAS colorectal cancer (CRC) cells release protein-laden exosomes that can alter the tumor microenvironment. To test whether exosomal RNAs also contribute to changes in gene expression in recipient cells, and whether mutant KRAS might regulate the composition of secreted microRNAs (miRNAs), we compared small RNAs of cells and matched exosomes from isogenic CRC cell lines differing only in KRAS status. We show that exosomal profiles are distinct from cellular profiles, and mutant exosomes cluster separately from wild-type KRAS exosomes. miR-10b was selectively increased in wild-type exosomes, while miR-100 was increased in mutant exosomes. Neutral sphingomyelinase inhibition caused accumulation of miR-100 only in mutant cells, suggesting KRAS-dependent miRNA export. In Transwell co-culture experiments, mutant donor cells conferred miR-100-mediated target repression in wild-type-recipient cells. These findings suggest that extracellular miRNAs can function in target cells and uncover a potential new mode of action for mutant KRAS in CRC.

DOI: 10.7554/eLife.07197.001

\section{Introduction}

An emerging paradigm in the study of cell signaling is the potential role for post-transcriptional gene regulation by extracellular RNAs. microRNAs (miRNAs) are perhaps the best characterized class of small noncoding RNAs (ncRNAs) that have been detected in extracellular fluids (Valadi et al., 2007). Mature miRNAs are 21-23 nucleotides in length and bind to target mRNAs to inhibit their expression (Krol et al., 2010). Because miRNAs imperfectly pair with their mRNA targets, they can potentially regulate hundreds of transcripts within a genome (Bartel and Chen, 2004). However, individual miRNAs exhibit exquisite tissue-specific patterns of expression (Wienholds et al., 2005), control cell fate decisions (Alvarez-Garcia and Miska, 2005), and are often aberrantly expressed in human cancers (Thomson et al., 2006), affording possible disease-specific signatures with diagnostic, prognostic, and therapeutic potential (Lu et al., 2005; Volinia et al., 2006). 
eLife digest Cells use several different methods to control which genes are expressed to produce the proteins and RNA molecules that they need to work efficiently. The first step of gene expression is to transcribe a gene to form an RNA molecule. Protein-coding mRNA molecules can then be translated to make proteins. However, many RNA transcripts do not encode proteins. One example of these non-coding RNAs is a class of small RNAs called microRNAs (miRNAs), which are predicted to target more than $60 \%$ of protein-coding genes and can control which proteins are made.

It was once thought that miRNAs exist only within the cell where they are synthesized. Recently, however, miRNAs have been found outside the cell bound to lipids and proteins, or encased in extracellular vesicles, such as exosomes. Exosomes are small bubble-like structures used by cells to export material into the space outside of cells. Exosomes containing miRNAs can circulate throughout the body, potentially transferring information between cells to alter gene expression in recipient cells.

Many colorectal cancer cells have mutations in a gene that encodes a protein called KRAS. In 2011 and 2013, researchers found that the contents of the exosomes released from these mutant KRAS colorectal cancer cells can influence normal cells in ways that would help a cancer to spread.

Furthermore, the exosomes released from the KRAS mutant cells contain different proteins than nonmutant cells.

Now, Cha, Franklin et al.-including several researchers who worked on the 2011 and 2013 studies-show that exosomes released by mutant KRAS cells also contain miRNAs, and that these miRNAs are different from the ones exported in exosomes by cells with a normal copy of the KRAS gene. In particular, several miRNAs that suppress cancer growth in a healthy cell are found at lower levels in mutant KRAS cells. Instead, these miRNAs are highly represented in the exosomes that are released by the KRAS mutant cells.

When cells with a normal copy of the KRAS gene were exposed to the contents of the exosomes released from KRAS mutant cells, an important gene involved in cell growth was suppressed. This indicates that the miRNAs exported from cancerous cells can influence gene expression in neighboring cells. Getting rid of such cancer-suppressing miRNAs could give cancer cells a growth advantage over normal cells to promote tumor growth. Cha, Franklin et al. also suggest that it might be possible to create a non-invasive test to detect colorectal cancer by monitoring the levels of circulating miRNAs in patients. Potential treatments for the disease could also target these miRNAs. DOI: 10.7554/eLife.07197.002

In addition to their intracellular roles, recent experiments have identified miRNAs outside the cell in extracellular vesicles (EVs) including exosomes or larger vesicles (Valadi et alı, 2007; Crescitelli et alı, 2013), in high-density lipoprotein particles (Vickers et al., 2011), or in smaller complexes with Argonaute 2 protein (Arroyo et alo, 2011). Exosomes are small 40-130 nm vesicles of endosomal origin that are secreted by all cells and can fuse and be internalized by recipient cells (Valadi et al., 2007; Kosaka et al., 2010; Higginbotham et al., 2011; Mittelbrunn et al., 2011; Montecalvo et al., 2012). It has been suggested that protein cargo transfer by exosomes between cells is associated with tumor aggressiveness and metastasis (Skog et al., 2008; Higginbotham et al., 2011; Luga et al., 2012; Hoshino et alo, 2013; Costa-Silva et al., 2015). With the discovery that miRNAs and other RNAs can also be packaged into EVs, or exported by other extracellular mechanisms, it remains unclear the extent to which RNA cargo is sorted for export and how it is dysregulated in disease conditions, such as cancer.

Despite accumulating evidence that exosomes are biologically active, little is known regarding how oncogenic signaling affects the repertoire of miRNAs or proteins that are selected for secretion. Given the potential of cancer-derived secreted RNAs to modulate the tumor microenvironment, elucidation of the potential mechanisms for selective sorting of cargo into exosomes is critical to understanding extracellular signaling by RNA. KRAS mutations occur in approximately $34-45 \%$ of colon cancers (Wong and Cunningham, 2008). We have previously shown that exosomes from mutant KRAS colorectal cancer (CRC) cells can be transferred to wild-type cells to induce cell growth and migration (Higginbotham et al., 2011; Demory Beckler et al., 2013). Compared to exosomes derived from 
isogenically matched wild-type cells, exosomes derived from mutant KRAS cells contain dramatically different protein cargo (Demory Beckler et al., 2013). Here, we show that KRAS status also prominently affects the miRNA profile in cells and their corresponding exosomes. Exosomal miRNA profiles are distinct from cellular miRNA patterns, and exosomal miRNA profiles are better predictors of KRAS status than cellular miRNA profiles. Furthermore, we show that cellular trafficking of miRNAs is sensitive to neutral sphingomyelinase (nSMase) inhibition in mutant, but not wild type, KRAS cells and that transfer of miRNAs between cells can functionally alter gene expression in recipient cells.

\section{Results}

\section{Small ncRNAs are differentially distributed in exosomes}

Because small RNAs are thought to be sorted at endosomal membranes and since KRAS signaling can also occur on late endosomes (Lu et al., 2005), we hypothesized that oncogenic KRAS signaling could alter RNA export into exosomes. We prepared small RNA libraries from both exosomes and whole cells using isogenically matched CRC cell lines that differ only in KRAS status (Figure 1-source data 1) (Shirasawa et al., 1993). Exosomes were purified using differential centrifugation and consisted of vesicles ranging in size from 40 to $130 \mathrm{~nm}$ (Higginbotham et al., 2011; Demory Beckler et al., 2013). These preparations exclude larger microvesicles but contain smaller lipoproteins and probably other small RNA-protein complexes (unpublished observation). Comprehensive sequencing analyses of both cellular and exosomal small RNAs from all three cell lines revealed that more than $85 \%$ of the reads from the cellular RNA libraries mapped to the genome, compared to only $50-71 \%$ from the exosomal libraries (Figure 1A). The non-mappable reads consisted largely of sequences that contain mismatches to genomic sequences.

The global small RNA profiles identified reads from various classes of RNA, including miRNAs, with differential enrichment of specific RNAs in both the cellular and exosomal fractions. Compared to cellular RNA samples, which displayed an enrichment of miRNA sequences ( $70 \%)$, miRNA reads in exosomal samples comprised a smaller percentage of the total small reads (5-18\%) compared to other ncRNA classes (e.g., tRNAs, rRNAs, snRNAs) (Figure 1B,C, Supplementary file 1). Most of these reads appear to be the fragments of larger RNAs, both cytoplasmic and nuclear. It is not clear how these RNAs are associated with and/or deposited into exosomes.

The size distribution of cellular small RNA matched that expected from miRNA-derived reads (21-23 nucleotides). However, the small RNA read distribution from exosomes was much broader with many reads smaller than 22 nucleotides in length (Figure 1-figure supplement 1). Given that these reads map to RNAs other than known miRNAs, these data suggest that a large proportion of small exosomal RNA reads is derived from processing of other RNAs, in addition to post-transcriptionally modified miRNA reads that are apparently subject to editing, trimming, and/or tailing (Koppers-Lalic et al., 2014). Consistent with this, when read identity was restricted to miRNAs by mapping back to known miRNA hairpin sequences, the length distribution of mappable reads was nearly identical between cells and exosomes (Figure 1D).

\section{miRNAs are differentially enriched in exosomes dependent on KRAS status}

Focusing on mappable reads, we sought to ascertain whether miRNAs might be differentially represented when comparing cells to their secreted exosomes. For this, we quantified the relative abundance of individual miRNAs and made pairwise comparisons between normalized miRNA reads. Spearman correlation analyses demonstrated high correlation between replicates of individual cell lines $(r=0.95-0.96)$ and between cellular data sets differing only in KRAS status ( $r=0.92-0.96)$ (Figure 2-figure supplements 1-3). In contrast, the miRNA profiles from exosomes compared to their parent cells were less correlated (DKO-1 $r=0.67-0.81$, DKs-8 $r=0.64-0.71$, DLD-1 $r=0.64-0.69$ ) (Figure 2-figure supplements 1, 2, 4).

We next utilized principal component (PC) analysis to determine whether the overall miRNA profiles could distinguish between cells and exosomes and/or between wild-type and mutant KRAS status. The miRNA profiles from the three cell lines all clustered close to one another indicating that overall miRNA expression profiles are fairly similar among the different cell types (Figure 2). In marked contrast, PC analysis revealed that exosomal miRNA profiles clearly segregate according to KRAS status (Figure 2). Relatively, minor differences between cellular miRNA expression profiles become 
A

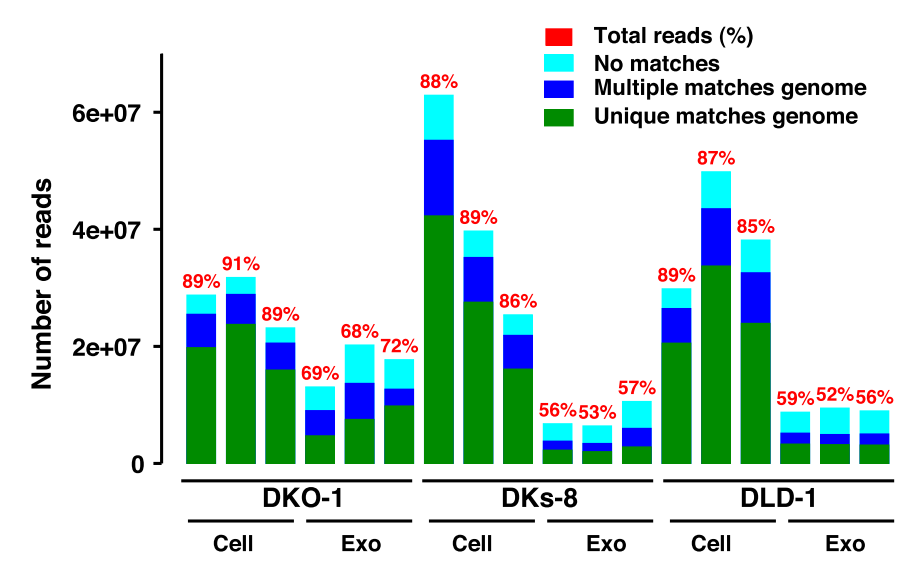

B

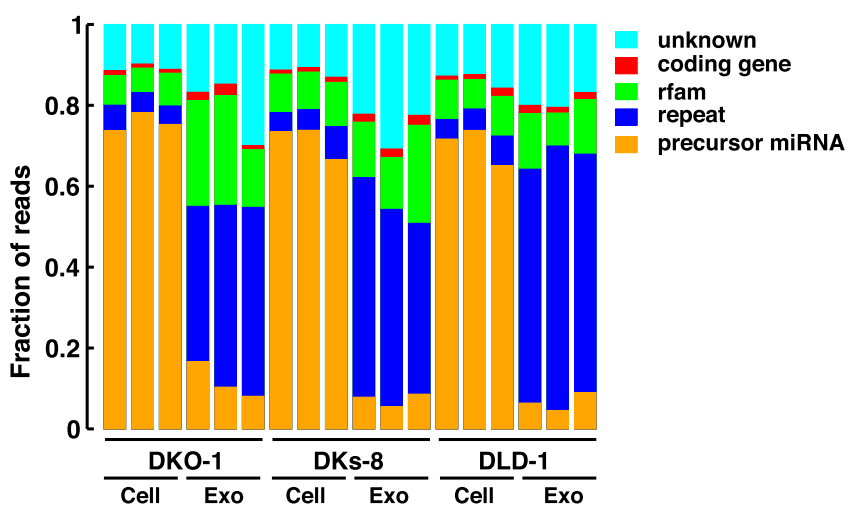

C

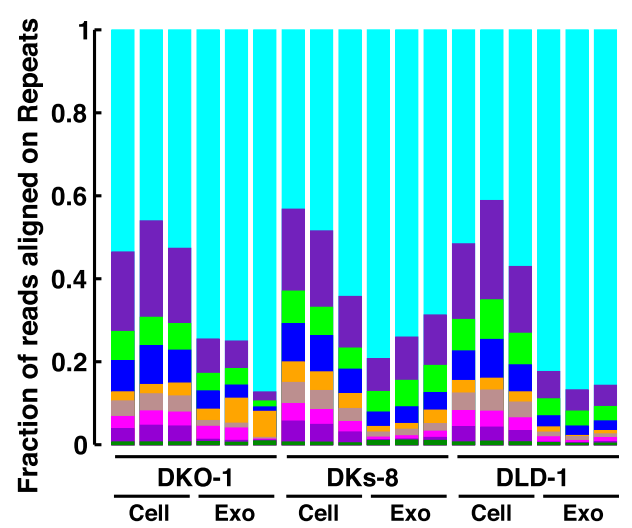

D
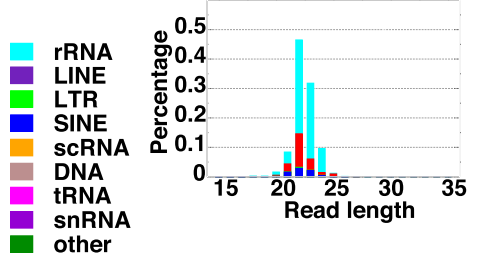

DKO-1 exo

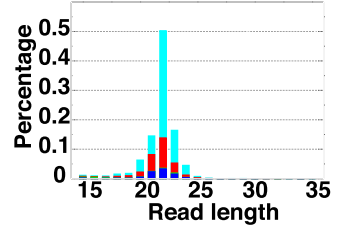

DKs-8 cell

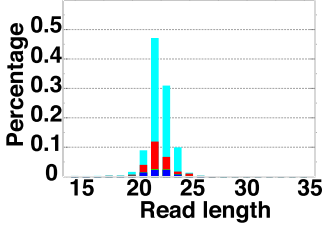

DKs-8 exo

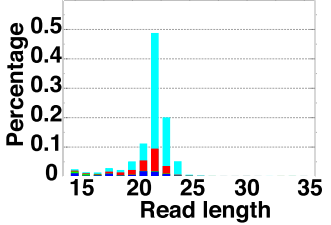

DLD-1 cell

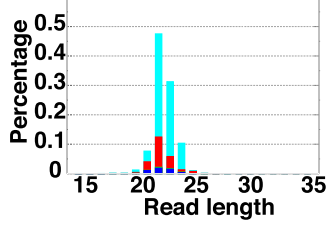

DLD-1 exo

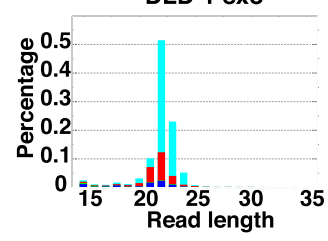

Figure 1. Small RNA sequencing analysis of cellular and exosomal RNAs from CRC cell lines. Shown are (A) total read numbers (y-axis) and the total percentage of mappable reads (red), percentage of unique mappable reads (green), reads that map to multiple genomic locations (dark blue), and those that could not be mapped (cyan). (B) The majority of mappable small RNA reads were derived from noncoding RNAs in cells and exosomes. In cells, the majority of small RNA reads mapped to microRNAs (miRNAs) (miRbase 19), whereas in exosomes, the majority of small RNA reads mapped to repetitive elements. (C) The origin of repetitive reads from exosomal small RNA sequencing is shown. Repeat reads were annotated based on RepeatMasker and Rfam classified into tRNAs, rRNAs, snRNAs, and others. (D) The length distribution of reads mapping to miRNA hairpins was determined for small RNA reads from the three $C R C$ cell lines and their purified exosomes. Colors represent the nucleotides identified for the $5^{\prime}$ base, $T$ (cyan), $A$ (red), $G$ (green), and C (blue). Figure 1-figure supplement 1.

DOI: 10.7554/eLife.07197.003

The following source data and figure supplement are available for figure 1:

Source data 1. Colorectal cancer (CRC) cell lines.

DOI: 10.7554/eLife.07197.004

Figure supplement 1. Length distribution of small RNA reads to genome.

DOI: 10.7554/eLife.07197.005

much more prominent when comparing exosomal miRNA patterns (Figure 2-figure supplement 3). This indicates that the presence of a mutant KRAS allele alters sorting of specific miRNAs to exosomes, a finding that has potentially important implications for biomarker development.

To gain more insight into the relative abundance of miRNAs in cells vs matched exosomes, we examined the most abundant miRNA species in the various sequencing libraries (determined by mean reads of individual miRNAs). For many miRNAs, exosomal abundance correlated with cellular abundance (Supplementary file 2). However, calculation of fold changes among the three isogenic KRAS cell lines, and exosomes released from these cells, showed that distinct subsets of miRNAs are 


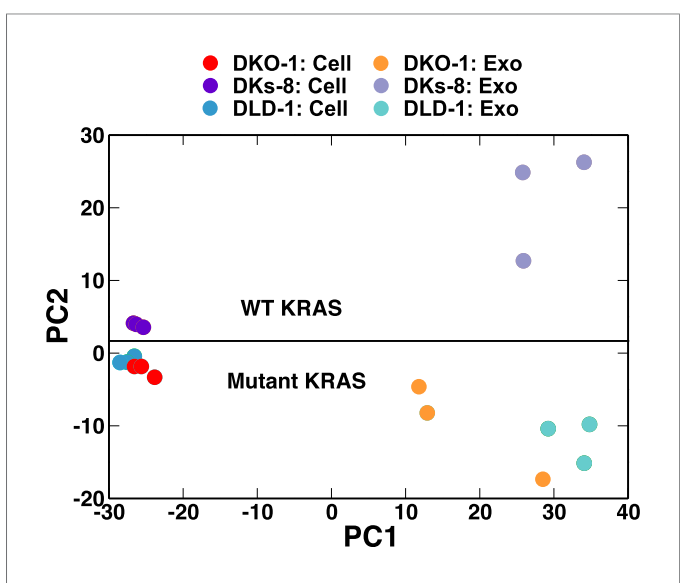

Figure 2. Small RNA composition segregates with KRAS status. Principal component analysis was performed comparing small RNA sequencing data sets from CRC cells and exosomes. The small RNA composition from cells differed significantly from exosomes. Nevertheless, clustering showed that mutant KRAS status could be

Figure 2-figure supplements 1-4.

DOI: 10.7554/eLife.07197.006

The following figure supplements are available for figure 2:

Figure supplement 1. Spearman correlations between miRNA expression profiles in cells and exosomes. DOI: 10.7554/eLife.07197.007

Figure supplement 2. Spearman correlations between miRNA expression profiles in cells and exosomes. DOI: 10.7554/eLife.07197.008

Figure supplement 3. Spearman correlations between miRNA expression profiles in cells (top) and exosomes (bottoms) in reads per million (RPM).

DOI: 10.7554/eLife.07197.009

Figure supplement 4. Spearman correlations comparing miRNA expression profiles in exosomes to parent cell in RPM.

DOI: 10.7554/eLife.07197.010 inferred from small RNA composition. Also see

enriched in either exosomes or cells (Tables 1, 2). For all three cell lines, 25 miRNAs were consistently upregulated in cells and 29 miRNAs were consistently upregulated in exosomes (Figure 3A,B). Additionally, the diversity of miRNAs was substantially greater in mutant KRAS DKO-1 exosomes (94 unique miRNAs) compared to parental DLD-1 or wild-type KRAS DKs-8 exosomes (Figure 3B). A select subset of cell and exosomally targeted miRNAs were validated separately by quantitative reversetranscription PCR (qRT/PCR) (Figure 3C). Collectively, these data indicate that the miRNA profiles observed in exosomes are distinct from their parental cells with specific miRNAs preferentially overrepresented or underrepresented in exosomes. We observed a mutant KRAS-specific pattern of secreted miRNAs, consistent with the hypothesis that dysregulation of miRNA metabolism is associated with tumorigenesis, a previously unrecognized feature of mutant KRAS.

\section{KRAS-dependent sorting of miRNAs}

miR-100

Down regulation of miR-100-5p was observed in mutant KRAS DKO-1 and parental DLD-1 cells compared to wild-type KRAS DKs-8 cells (Table 1). This is consistent with reports that have shown decreased miR-100 expression in metastatic cancers (Petrelli et al., 2012; Gebeshuber and Martinez, 2013). miR-100 has also been shown to negatively regulate migration, invasion, and the epithelial-mesenchymal transition (EMT) (Chen et al., 2014; Wang et al., 2014; Zhou et al., 2015). Interestingly, miR-100 was enriched in exosomes derived from mutant KRAS cells (>eightfold and >threefold enriched in DKO-1 and DLD-1 exosomes, respectively; Supplementary file 2), suggesting that decrease of miR-100 in cells is due to secretion in exosomes. This is in line with findings that circulating levels of miR-100 are upregulated in the plasma of mutant KRAS-expressing mouse pancreatic cancer models and in patients with pancreatic cancer (LaConti et al., 2011). More broadly, the observation that miR$100-5 p$ specifically accumulates in exosomes suggests that there may be sequence-specific requirements for the sorting of certain miRNAs into exosomes.

miR-10b

Our RNA sequencing data identified miR-10b as preferentially secreted in exosomes isolated from cells harboring a wild-type KRAS allele (>threefold-change and >twofold-change enrichment in DKs-8 and DLD-1 exosomes, respectively) but retained in mutant KRAS DKO-1 cells ( threefold-change cell enrichment). miR-10b is referred to as an oncomiR because it is frequently upregulated during progression of various cancers, including CRC (Ma, 2010).

$\operatorname{miR}-320$

miR-320 is aberrantly expressed in several types of cancer, including colon cancer. It is expressed in the proliferative compartment of normal colonic crypts (Schepeler et al., 2008; Hsieh et al., 2013). 
Table 1. Differential expression of miRNAs in colorectal cancer cells* DKO-1

\begin{tabular}{|c|c|c|c|}
\hline hsa-miR-548u & hsa-miR-16-1-3p & hsa-miR-33a-3p & hsa-miR-33a-5p \\
\hline hsa-miR-31-5p & hsa-miR-181b-3p & hsa-miR-450a-5p & hsa-miR-424-5p \\
\hline hsa-miR-9-5p & hsa-miR-219-5p & hsa-miR-190a & hsa-miR-573 \\
\hline hsa-miR-30d-3p & hsa-miR-204-5p & hsa-miR-1226-3p & hsa-miR-499a-5p \\
\hline hsa-miR-450b-5p & hsa-miR-499b-3p & hsa-miR-3662 & hsa-miR-20a-3p \\
\hline hsa-miR-27b-5p & hsa-miR-5701 & hsa-miR-4677-3p & hsa-let-7i-5p \\
\hline hsa-miR-331-3p & hsa-miR-31-3p & hsa-miR-651 & hsa-miR-1306-5p \\
\hline hsa-miR-147b & hsa-miR-3611 & hsa-miR-1305 & hsa-miR-148a-3p \\
\hline hsa-miR-27b-3p & hsa-miR-1306-3p & hsa-miR-374b-3p & hsa-miR-1260b \\
\hline hsa-miR-3940-3p & hsa-miR-200c-5p & hsa-miR-548ar-3p & \\
\hline \multicolumn{4}{|l|}{ DKs-8 } \\
\hline hsa-miR-132-5p & hsa-miR-484 & hsa-miR-374a-5p & hsa-miR-1180 \\
\hline hsa-miR-1307-3p & hsa-miR-200a-5p & hsa-miR-548o-3p & hsa-miR-149-5p \\
\hline hsa-miR-3615 & hsa-miR-100-5p & hsa-miR-197-3p & hsa-miR-378a-5p \\
\hline \multicolumn{4}{|l|}{ hsa-let-7a-3p } \\
\hline \multicolumn{4}{|l|}{ DLD-1 } \\
\hline hsa-miR-141-3p & hsa-miR-26b-5p & hsa-miR-24-3p & hsa-miR-3074-5p \\
\hline hsa-miR-15a-5p & hsa-miR-27a-3p & hsa-miR-3613-5p & hsa-miR-30b-5p \\
\hline hsa-miR-29a-3p & hsa-miR-301a-5p & hsa-let-7i-3p & hsa-miR-185-5p \\
\hline hsa-let-7g-5p & hsa-miR-23b-3p & hsa-miR-22-3p & \\
\hline \multicolumn{4}{|l|}{ DKO-1 and DKs-8 } \\
\hline hsa-miR-141-5p & hsa-miR-582-5p & & \\
\hline \multicolumn{4}{|l|}{ DKO-1 and DLD-1 } \\
\hline hsa-miR-556-3p & hsa-miR-374a-3p & hsa-miR-106b-5p & hsa-miR-17-3p \\
\hline hsa-miR-24-1-5p & hsa-miR-340-3p & & \\
\hline \multicolumn{4}{|l|}{ DLD-1 and DKs-8 } \\
\hline hsa-miR-24-2-5p & hsa-miR-106a-5p & hsa-miR-30e-5p & hsa-miR-107 \\
\hline hsa-miR-429 & hsa-miR-98-5p & hsa-miR-425-5p & hsa-miR-140-5p \\
\hline hsa-miR-93-5p & hsa-miR-210 & hsa-miR-126-3p & hsa-miR-194-5p \\
\hline hsa-miR-29b-3p & hsa-miR-15b-5p & hsa-miR-362-5p & hsa-miR-27a-5p \\
\hline hsa-miR-454-3p & hsa-miR-452-5p & hsa-miR-196b-5p & \\
\hline \multicolumn{4}{|c|}{ DKO-1, DLD-1 and DKs-8 } \\
\hline hsa-miR-32-5p & hsa-miR-582-3p & hsa-miR-542-3p & hsa-miR-96-5p \\
\hline hsa-miR-101-3p & hsa-miR-18a-5p & hsa-miR-3529-3p & hsa-miR-7-5p \\
\hline hsa-miR-19a-3p & hsa-miR-142-3p & hsa-miR-20a-5p & hsa-miR-32-3p \\
\hline hsa-miR-130b-5p & hsa-miR-1278 & hsa-miR-7-1-3p & hsa-miR-590-3p \\
\hline hsa-miR-4473 & hsa-miR-17-5p & hsa-miR-103a-3p & hsa-miR-103b \\
\hline hsa-miR-19b-3p & hsa-miR-340-5p & hsa-miR-200a-3p & hsa-miR-34a-5p \\
\hline \multicolumn{4}{|l|}{ hsa-miR-372 } \\
\hline $\begin{array}{l}{ }^{*} \text { miRNAs differentia } \\
\text { miRNAs expression } \\
\text { were enriched in jus } \\
\text { were identified that } \\
\text { were unique to DKC } \\
\text { DOI: } 10.7554 / \text { Life.C }\end{array}$ & d in all three cell ty & $\begin{array}{l}\text { eads in exosomes } \\
\text {-1, and DLD-1 cel } \\
\text { ed between combir } \\
\text { enriched in DKs-8 }\end{array}$ & $\begin{array}{l}\text { ere identified that } \\
\text { s. For miRNAs, } 25 \\
\text { LD-1 cells, and } 39\end{array}$ \\
\hline
\end{tabular}


Table 2. Differential distribution of miRNAs in exosomes* DKO-1

\begin{tabular}{|c|c|c|c|}
\hline hsa-miR-139-5p & hsa-miR-3178 & hsa-miR-151b & hsa-miR-125b-1-3p \\
\hline hsa-miR-193b-3p & hsa-miR-935 & hsa-miR-130b-3p & hsa-miR-628-3p \\
\hline hsa-miR-139-3p & hsa-let-7d-3p & hsa-miR-589-3p & hsa-miR-4532 \\
\hline hsa-miR-451a & hsa-miR-6087 & hsa-miR-151a-5p & hsa-miR-940 \\
\hline hsa-miR-222-3p & hsa-miR-766-5p & hsa-miR-505-5p & hsa-miR-3187-3p \\
\hline hsa-miR-125a-3p & hsa-miR-3679-5p & hsa-miR-4436b-3p & hsa-miR-4787-3p \\
\hline hsa-miR-2277-3p & hsa-miR-361-5p & hsa-miR-1293 & hsa-miR-3183 \\
\hline hsa-miR-3162-5p & hsa-miR-642a-3p & hsa-miR-642b-5p & hsa-miR-197-5p \\
\hline hsa-miR-324-3p & hsa-miR-145-3p & hsa-miR-3182 & hsa-miR-3127-3p \\
\hline hsa-miR-3127-5p & hsa-miR-4728-3p & hsa-miR-3184-5p & hsa-miR-125b-5p \\
\hline hsa-miR-186-5p & hsa-miR-1 & hsa-miR-100-5p & hsa-miR-423-3p \\
\hline hsa-miR-766-3p & hsa-miR-4753-5p & hsa-miR-145-5p & hsa-miR-4724-5p \\
\hline hsa-miR-373-3p & hsa-miR-223-5p & hsa-miR-1307-5p & hsa-miR-1914-3p \\
\hline hsa-miR-3121-3p & hsa-miR-3613-3p & hsa-miR-205-5p & hsa-miR-98-3p \\
\hline hsa-miR-23a-3p & hsa-miR-3124-5p & hsa-miR-3656 & hsa-miR-3918 \\
\hline hsa-miR-4449 & hsa-miR-378c & hsa-miR-3138 & hsa-miR-1910 \\
\hline hsa-miR-3174 & hsa-miR-4466 & hsa-miR-3679-3p & hsa-miR-3200-5p \\
\hline hsa-miR-6511b-5p & hsa-miR-1247-5p & hsa-miR-22-3p & hsa-miR-877-5p \\
\hline hsa-miR-4687-3p & hsa-miR-1292-5p & hsa-miR-181c-5p & hsa-miR-6131 \\
\hline hsa-miR-6513-5p & hsa-miR-3661 & hsa-miR-132-3p & hsa-miR-214-3p \\
\hline hsa-miR-574-3p & hsa-miR-3190-3p & hsa-miR-326 & hsa-miR-3191-5p \\
\hline hsa-miR-3198 & hsa-miR-3928 & hsa-miR-629-3p & hsa-miR-4489 \\
\hline hsa-miR-4700-5p & hsa-miR-5006-5p & hsa-miR-5088 & hsa-miR-2110 \\
\hline hsa-miR-3911 & hsa-miR-3146 & & \\
\hline \multicolumn{4}{|l|}{ DKs-8 } \\
\hline hsa-miR-1224-5p & hsa-let-7b-5p & hsa-miR-155-5p & hsa-let-7c \\
\hline hsa-let-7a-5p & hsa-miR-146b-5p & hsa-miR-4647 & hsa-miR-4494 \\
\hline hsa-miR-711 & hsa-miR-1263 & & \\
\hline \multicolumn{4}{|l|}{ DLD-1 } \\
\hline hsa-miR-1226-5p & hsa-miR-4745-5p & hsa-miR-4435 & hsa-miR-939-5p \\
\hline hsa-miR-409-3p & hsa-miR-1304-3p & & \\
\hline \multicolumn{4}{|l|}{ DKO-1 and DKs-8 } \\
\hline hsa-miR-146a-5p & hsa-miR-4508 & hsa-miR-224-5p & hsa-miR-4429 \\
\hline hsa-miR-222-5p & hsa-miR-629-5p & hsa-miR-4492 & hsa-miR-3653 \\
\hline hsa-miR-320a & hsa-miR-1290 & hsa-miR-1262 & hsa-miR-5010-5p \\
\hline hsa-miR-204-3p & hsa-miR-4461 & hsa-miR-5187-5p & \\
\hline \multicolumn{4}{|l|}{ DKO-1 and DLD-1 } \\
\hline hsa-miR-483-5p & hsa-miR-4658 & hsa-miR-4758-5p & hsa-miR-492 \\
\hline hsa-miR-5001-5p & hsa-miR-371a-5p & hsa-miR-1323 & hsa-miR-371b-3p \\
\hline hsa-miR-501-3p & hsa-miR-4446-3p & hsa-miR-6511a-5p & hsa-miR-30a-3p \\
\hline \multicolumn{4}{|l|}{ hsa-miR-4727-3p } \\
\hline \multicolumn{4}{|l|}{ DLD-1 and DKs-8 } \\
\hline hsa-miR-28-3p & hsa-miR-3934-5p & & \\
\hline
\end{tabular}

Table 2. Continued on next page 
Table 2. Continued

\begin{tabular}{|c|c|c|c|}
\hline hsa-miR-658 & hsa-miR-320d & hsa-miR-4792 & hsa-miR-1246 \\
\hline hsa-miR-320e & hsa-miR-4516 & hsa-miR-320b & hsa-miR-4488 \\
\hline hsa-miR-1291 & hsa-miR-320c & hsa-miR-4634 & hsa-miR-3605-5p \\
\hline hsa-miR-4741 & hsa-miR-3591-3p & hsa-miR-122-5p & hsa-miR-486-3p \\
\hline hsa-miR-184 & hsa-miR-223-3p & hsa-miR-3651 & hsa-miR-486-5p \\
\hline hsa-miR-3180 & hsa-miR-3180-3p & hsa-miR-3168 & hsa-miR-4497 \\
\hline hsa-miR-423-5p & hsa-miR-3184-3p & hsa-miR-150-5p & hsa-miR-664a-5p \\
\hline \multicolumn{4}{|l|}{ hsa-miR-182-5p } \\
\hline \multicolumn{4}{|c|}{$\begin{array}{l}\text { *miRNAs differentially enriched in exosomes when comparing mean reads in exosomes vs cell. } \\
\text { miRNAs expression patterns were compared between exosomes purified from DKs-8, DKO-1, and DLD-1 cells. } \\
\text { miRNAs were identified that were enriched in exosomes from just one of the three cell lines or that overlapped } \\
\text { between combinations of cell lines. } 29 \text { miRNAs were common between exosomes from all three cell lines. } 94 \text { were } \\
\text { enriched in exosomes from DKO-1 cells, } 10 \text { in exosomes from DKs-8 cells, and only } 6 \text { in exosomes from DLD-1 cells } \\
\text { DOI: } 10.7554 / \text { eLife.07197.012 }\end{array}$} \\
\hline
\end{tabular}

miR-320 members (miR-320b, -c, d, and -e) were abundant in both mutant KRAS (DKO-1) and wildtype KRAS (DKs-8) exosomes, but underrepresented in the matched cells, indicating that some miRNAs are transcribed and predominantly exported into exosomes, independent of KRAS status (Table 2, Supplementary file 1A). Of these family members, miR-320a and miR-320b were the most abundant species represented in exosomes by our RNA sequencing analyses (miR-320a in DKO-1 exosomes, and miR-320b in DKs-8 and DKO-1 exosomes). Interestingly, however, we observed the largest enrichment for miR-320d (fold changes $>241$ in DKs-8 and $>229$ in DKO-1) in exosomes relative to cells, despite being fourfold less abundant than miR-320b levels. Because the $3^{\prime}$-terminus may be important in regulating miRNA stability and turnover, coupled to the fact that the sequences of miR-320a-d members differ only at their $3^{\prime}$-termini, enrichment of certain miRNAs in exosomes could be due to higher turnover/decay rates in cells.

\section{Exosomal secretion and strand selection}

Because we observed differential export of specific miRNAs, we investigated whether there might be miRNA sequence-specific sorting signals. Previous reports have shown differential accumulation of $5 p$ or $3 p$ strands in exosomes compared to parental cells (Ji et al., 2014). Thus, we analyzed our data sets to test whether exosomes might be preferentially enriched for one strand over the other. We were able to identify individual miRNAs where the two strands differentially sorted between cells and exosomes. For example, the $-5 p$ strands of miR-423 were overrepresented in DKO-1 exosomes but in exosomes from DKs-8 cells, both strands were overrepresented compared to cells (data not shown). This indicates that KRAS status may differentially affect selection of passenger or guide strands for sorting into exosomes for select individual miRNAs.

Individual miRNAs often exist as populations of variants (isomiRs) that differ in length and/or nucleotide composition generated by template- or non-template-directed variation (Burroughs et al., 2010; Newman et al., 2011; Neilsen et al., 2012). When we analyzed our sequencing data sets, we did not detect differential accumulation of isomers with variable $5^{\prime}$ termini (data not shown). For cellular miRNAs, most reads were full length with a slight enrichment in 3' non-templated addition of A-tailed miRNAs, regardless of KRAS status (Figure 4; Figure 4-figure supplement 1). For exosomes, we observed a slight enrichment for $C$ residues added to the $3^{\prime}$ ends of miRNAs from wild-type KRAS cells (Figure 4-figure supplement 1). We did not observe this in mutant KRAS exosomes, where instead, we noticed an increase in 3' trimming of miRNAs (Figure 4, Figure 4-figure supplement 2). Overall, it remains to be determined whether such modifications constitute a global exosomal sorting signal in these cells.

Consistent with published data, we have shown that miRNA expression patterns vary between parental cells and their cognate exosomes (Tables 1, 2, Figure 3A,B) (Valadi et al., 2007; Mittelbrunn et al., 2011; Ekstrom et al., 2012; Montecalvo et al., 2012; Squadrito et al., 2014). Differential export suggests that specific signals must exist to sort distinct miRNAs (Batagov et al., 
A

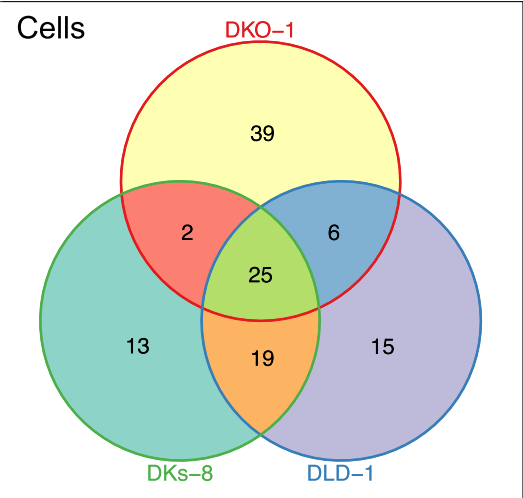

B

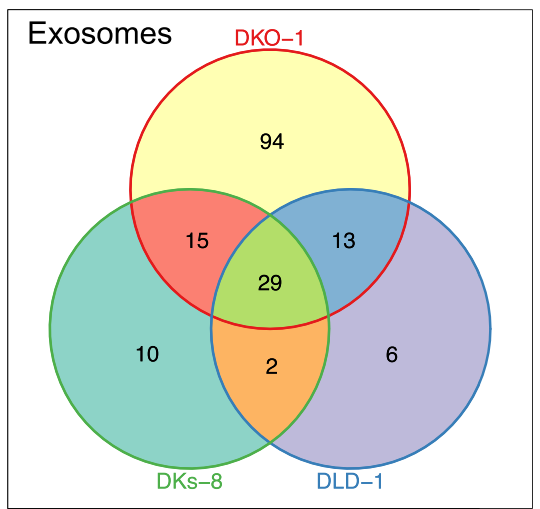

C

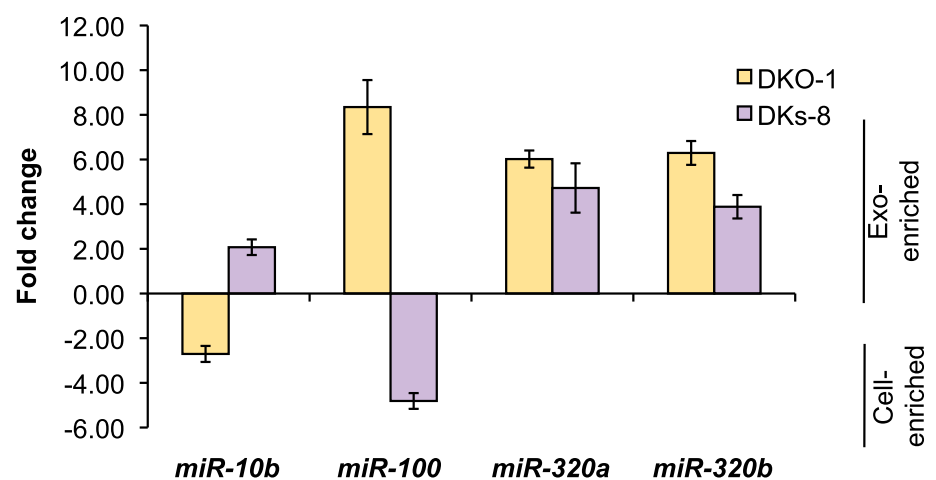

Figure 3. KRAS-dependent regulation of miRNAs in exosomes and cells. Differentially distributed miRNAs in (A) cells and (B) exosomes from the three CRC cell lines differing in KRAS status. (C) qRT-PCR validation of selected miRs from DKs-8 and DKO-1 cellular and exosomal RNA samples normalized to U6 snRNA. Fold changes were calculated using the $\Delta \Delta C(t)$ method comparing exosomes to cells. Negative fold changes indicate greater enrichment in cells, and positive fold changes indicate greater enrichment in exosomes. Also see Supplementary file 2.

DOI: 10.7554/eLife.07197.013

2011; Villarroya-Beltri et al., 2013). We therefore conducted MEME analysis to attempt to identify sequence motifs that might serve as targeting signals. When we examined all miRNA reads detected in exosomes, we did not find any global enrichment for specific sequences or motifs, including those reported to be bound by hnRNP A2B1 (GGAG or U/CC) (Bolukbasi et al., 2012; Villarroya-Beltri et al., 2013) (Figure 4-figure supplement 3). However, when we analyzed miR-320 because it is preferentially exported to exosomes independent of KRAS status, we were able to identify the GGAG sequence contained within the $3^{\prime}$ end of the mature sequence. Additionally, upon restricting our analysis to reads from the most differentially expressed miRNAs when comparing exosomes to cells, we found a slight enrichment for $C$ residues, possibly alternating $C$ residues in exosomal miRNAs (Figure 4-figure supplement 3).

\section{Sphingomyelinase-dependent sorting of miRNAs to exosomes}

Although little is understood regarding the molecular mechanisms for packaging exosomal miRNAs, recent evidence suggests that the secretion of miRNAs in exosomes is dependent on ceramide via its production by neutral sphingomyelinase 2 (nSMase 2) (Kosaka et al., 2010; Mittelbrunn et al., 2011). Inhibition of de novo ceramide synthesis by treatment with a nSMase inhibitor impaired exosomal miRNA release, apparently due to decreased formation of miRNA-containing exosomes 


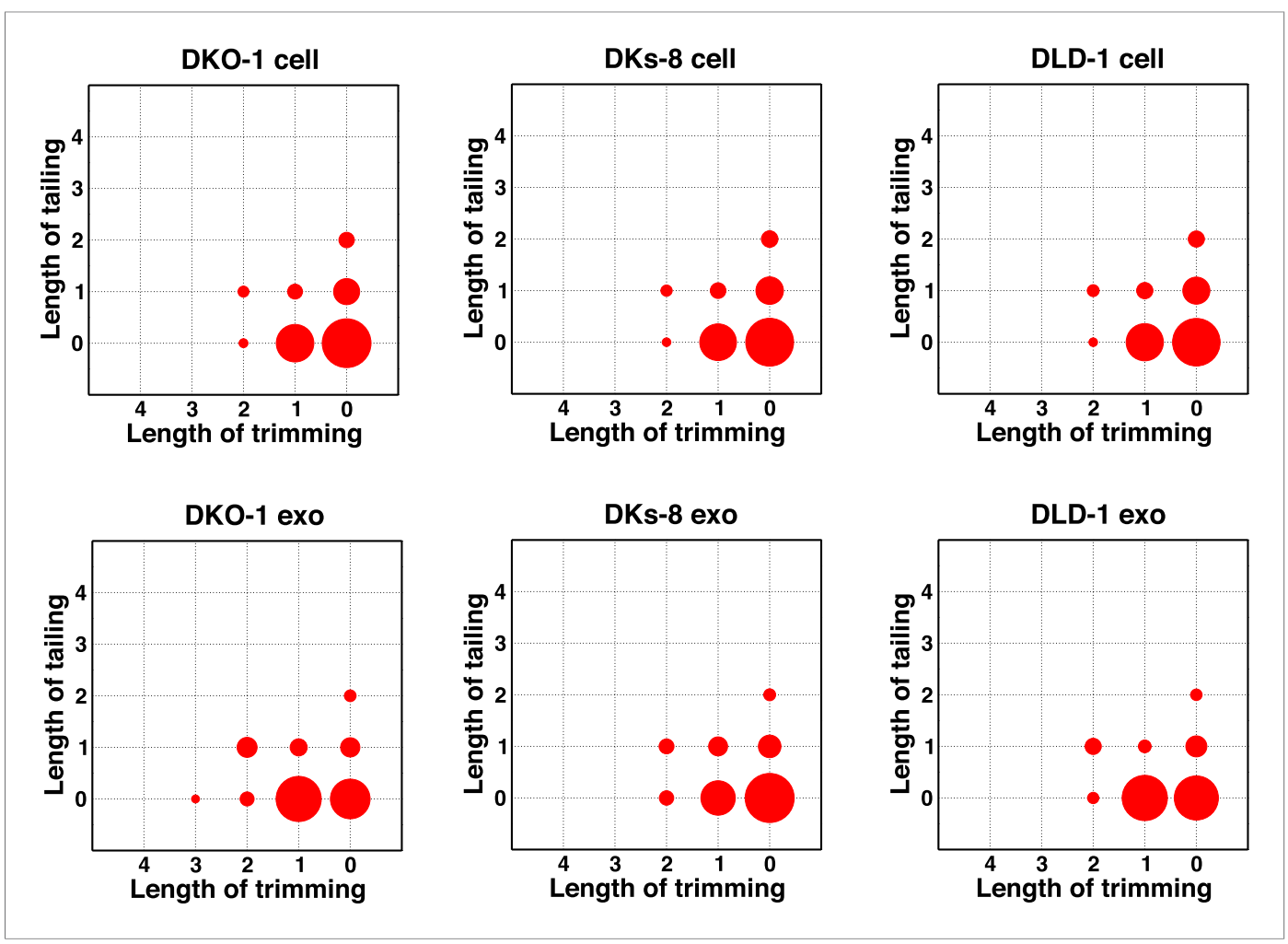

Figure 4. Comparison of miRNA $3^{\prime}$ trimming and tailing between cells and exosomes. Data from the heat maps shown in Figure 4-figure supplement 2 were pooled to illustrate overall changes in either 3' nucleotide additions (tailing) or 3' resection (trimming) compared to full length miRNA sequences (intact). Overall, the patterns between cells and between exosomes are very similar. A comparison of cells to exosomes shows that exosomes display a slight increase in trimmed miRNAs. Also see Figure 4-figure supplement 2.

DOI: 10.7554/eLife.07197.014

The following figure supplements are available for figure 4:

Figure supplement 1. Non-templated addition (NTA) of nucleotides to $3^{\prime}$ ends of miRNAs. DOI: 10.7554/eLife.07197.015

Figure supplement 2. Comparison of miRNA 3' trimming and tailing between cells and exosomes. DOI: 10.7554/eLife.07197.016

Figure supplement 3. MEME analysis of miRNA sequence in exosomes.

DOI: 10.7554/eLife.07197.017

(Kosaka et al., 2010; Mittelbrunn et al., 2011). To test the role of nSMase in miRNA secretion in our system, we treated CRC cells with the nSMase inhibitor, GW4869. We determined the effect of this inhibitor on miR-10b since it is preferentially found in wild-type KRAS DKs-8 exosomes, miR-100 since it is preferentially found in mutant KRAS DKO-1 and DLD-1 exosomes, and miR-320 which sorts into exosomes regardless of KRAS status. For miR-10b, we did not observe significant changes in its cellular levels after treatment with GW4869 in either wild-type KRAS DKs-8 or mutant KRAS DKO-1 cells (Figure $5 \mathrm{C}$ ). In contrast, inhibition of nSMase caused a $\sim$ threefold increase in intracellular levels of miR-100 in mutant KRAS DKO-1 cells but remained unchanged in wild-type DKs-8 KRAS cells (Figure 5A,B,C). Similarly, miR-320 levels were found to increase $(\sim 2.5$ fold) only in GW4869-treated mutant KRAS DKO-1 cells (Figure 5C). These data are most consistent with the hypothesis that impaired ceramide synthesis alters cellular accumulation of miRNAs dependent on mutant KRAS and suggest that multiple biogenic routes exist for miRNA secretion.

\section{Extracellular transfer of $\mathbf{m i R}-100$}

Several reports have found that extracellular miRNAs can be taken up by recipient cells to mediate heterotypic cell-cell interactions and facilitate target repression in neighboring cells 


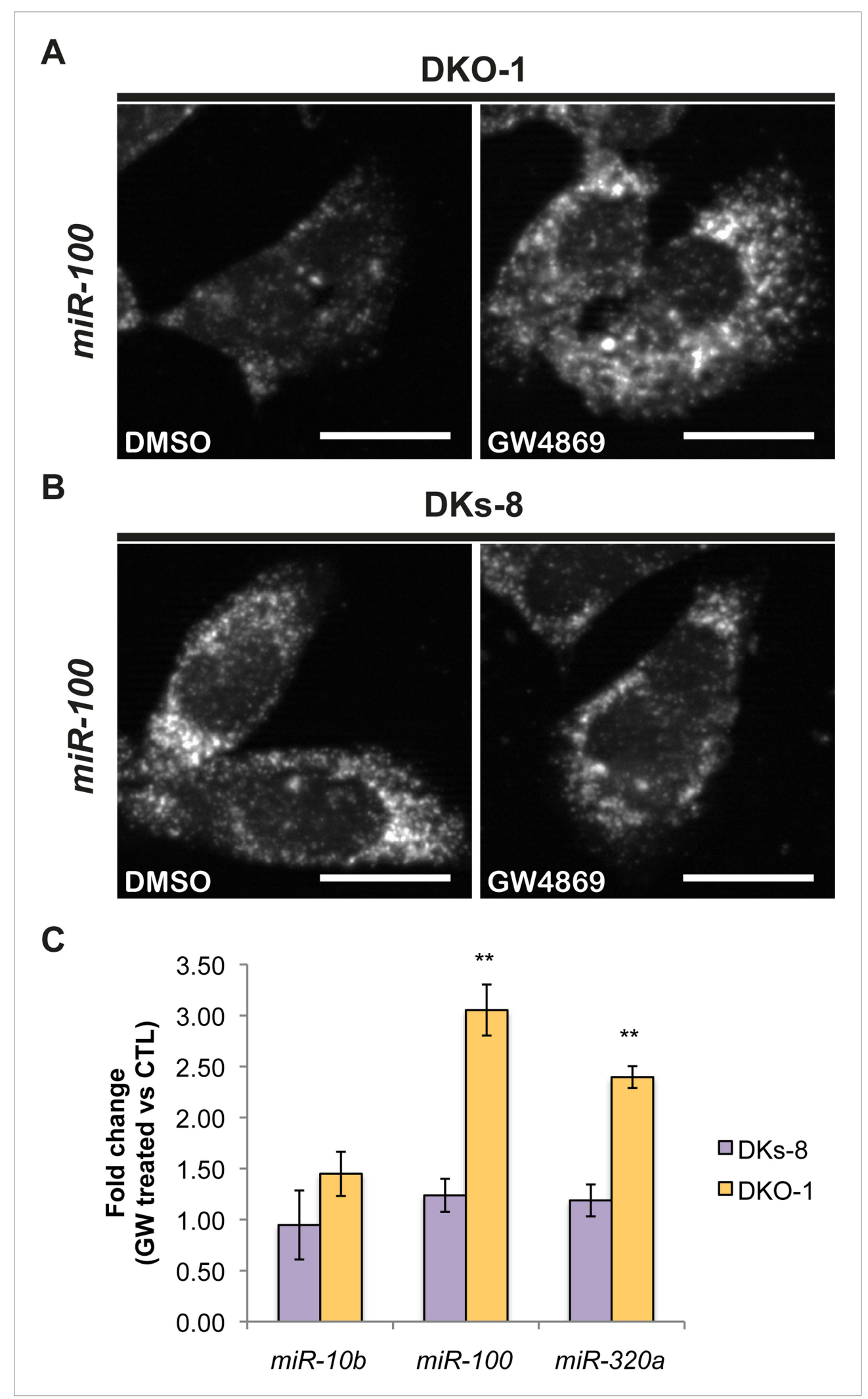

Figure 5. Ceramide-dependent miRNA export into exosomes. DKO-1 or DKs-8 cells were treated with an inhibitor of neutral sphingomyelinase 2 (nSMase 2), GW4869. After treatment, in situ hybridization experiments were performed with probes against miR-100 (A, B). (C) qRT-PCR for miR-10b, miR-100, and miR-320a was performed on Figure 5. continued on next page 
Figure 5. Continued

cells treated with GW4869 or DMSO, and fold change in expression was determined in treated vs untreated cells. In wild-type KRAS cells (DKs-8), inhibition of nSMase 2 had little or no effect on the cellular levels of these miRNAs. In contrast, mutant KRAS cells (DKO-1) showed an increase in cellular miRNA levels after inhibition of nSMAse 2. Data were derived from three biological replicates and performed in technical triplicates for qRT-PCR. Significance was determined by two-tailed, paired t-tests where * are $p$ values $\leq 0.05$ and ${ }^{* \star} \leq 0.01$.

DOI: 10.7554/eLife.07197.018

(Mittelbrunn et al., 2011; Boelens et al., 2014; Squadrito et al., 2014). To determine whether secreted miRNAs function in recipient cells, we designed luciferase (Luc) constructs containing either 3 perfect miR-100 recognition elements (MREs) in the $3^{\prime}$ untranslated region (UTR) (Luc-100-PT) or scrambled 3'UTR sequences that do not match any known miRNAs (Luc-CTL). These constructs were expressed in wild-type KRAS DKs-8 cells (recipient cells) in the presence or absence of donor cells. Baseline repression of Luc in the absence of donor cells was first analyzed to determine the levels of repression from endogenous miR-100 in DKs-8 cells. Compared to the scrambled control (Luc-CTL), strong Luc repression in the absence of donor cells was observed with perfect MREs (miR-100-PT) (Figure 6A). This supports our finding that miR-100 is expressed and retained in DKs-8 cells.

To determine whether secretion of miR-100 by mutant KRAS DKO-1 donor cells could further augment miR-100 function in recipient wild-type cells, Transwell co-culture experiments were performed with DKs-8 recipient cells expressing the Luc reporters in the presence of DKO-1 donor cells (Figure 6). Significantly increased repression of Luc was observed when the reporter construct containing three perfect miR-100 sites was used (miR-100-PT) (Figure 6A). Because exosomes released from DKO-1 cells contain abundant levels of miR-100, increased Luc repression is consistent with transfer of additional copies of miR-100. Two control experiments were performed to test the hypothesis that additional copies of miR-100 are transferred between donor and recipient cells. First, we treated donor cells with antagomirs that block production of miR-100. Luc repression was almost completely reversed upon pre-treatment of DKO-1 donor cells with a miR-100 hairpin antagomir inhibitor (Al-100) (Figure 6D). Second, we performed qRT/PCR to calculate the increase in miR-100 levels in recipient cells. Cells grown in the presence or absence of donor cells showed an approximate $34 \%$ increase in the levels of miR-100 (Figure 6E and Figure 6-figure supplement 2).

To further probe the repressive activity of miR-100, we performed co-culture experiments in which the recipient Dks-8 cells express Luc fused to the 3'UTR of mTOR, an endogenous miR-100 target (Nagaraja et al., 2010; Grundmann et al., 2011; Ge et al., 2014). As observed with miR-100-PT repression, Luc-mTOR was significantly repressed in the presence of mutant KRAS DKO-1 but not in the presence of wild-type KRAS DKs-8 donor cells (Figure 6B). This suggests that miR-100-repressive activity is specific to the presence of mutant KRAS DKO-1 donor cells. To confirm these results, we mutated the MREs within the mTOR 3'UTR and assayed for miR-100 activity (Figure 6-figure supplement 1). Mutation of individual sites did not show significantly different Luc repression (data not shown). However, upon mutation of two MREs (MS2), we observed a partial rescue of Luc expression (Figure 6C). This was further augmented upon mutation of all three sites (MS3), with a complete rescue of miR-100-mediated repressive activity (Figure 6C).

As a final test of miRNA transfer in the Transwell co-culture experiments, we created vectors expressing Luc fused to a 3'UTR containing perfect sites for miR-222 because miR-222 is not detectable in DKs-8-recipient cells, unlike miR-100. In this case, silencing of Luc should be due to transfer of miR-222 and not due to unforeseen changes in endogenous miRNA activity. We observed a greater than twofold repression of the miR-222 Luc reporter in recipient cells (Figure 6-figure supplement 3). These results support the hypothesis that miRNAs secreted by mutant KRAS cells can be transferred to recipient cells.

\section{Discussion}

In this study, we comprehensively examined the composition of small ncRNAs from exosomes and cells of isogenic CRC cell lines that differ only in KRAS status. By employing small RNA transcriptome analyses, we found that oncogenic KRAS selectively alters the miRNA profile in exosomes, and that ceramide depletion selectively promotes miRNA accumulation in mutant KRAS CRC cells. Distinct miRNA profiles between cells and their exosomes may be functionally coupled to mitogenic signaling. 
A

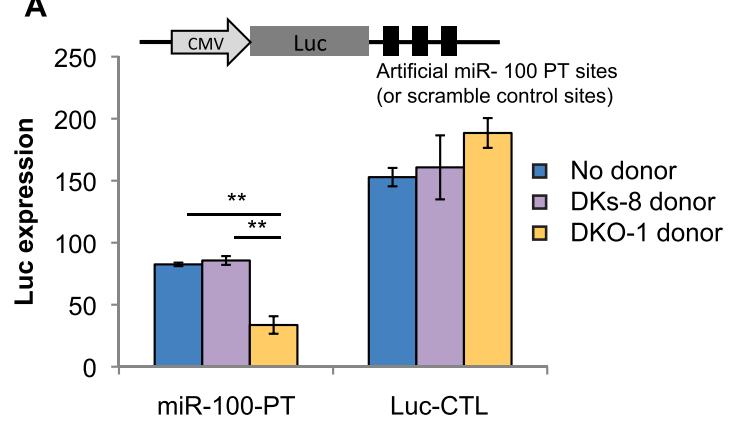

DKs-8 recipient

C

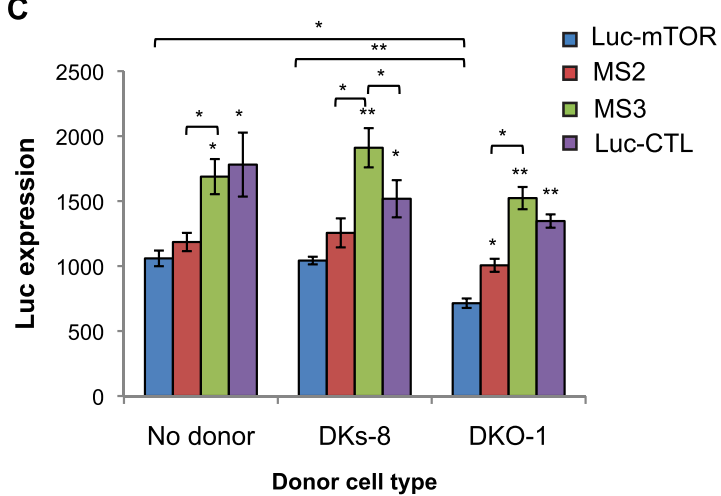

B

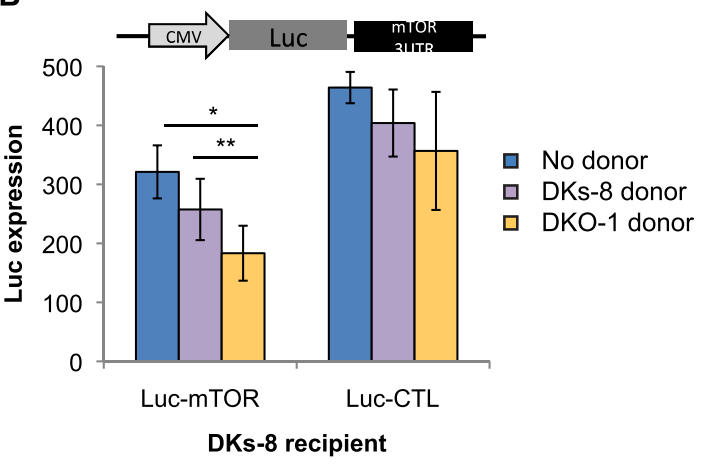

D

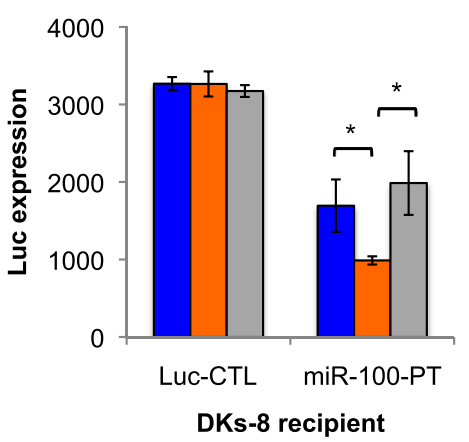

DKO-1 donor Al-100

口DKO-1 donor Al-ctl

$\square$ no donor

E

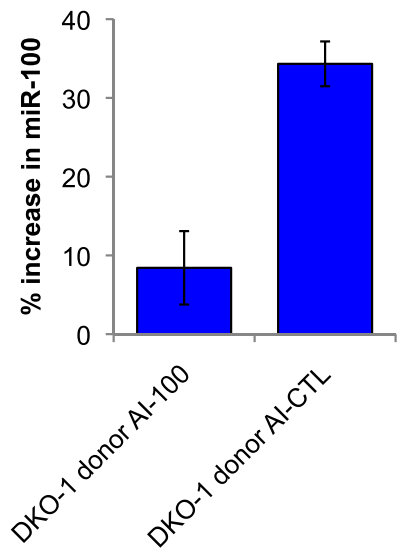

Figure 6. Transfer of extracellular miRNAs by mutant DKO-1 cells promotes target repression in wild-type DKs-8 cells. Transwell co-culture of DKs-8 recipient cells with or without DKs-8 or DKO-1 donor cells. Luciferase (Luc) expression was measured in DKs-8 recipient cells transiently expressing (A) Luc fused to three perfectly complementary synthetic miR-100 target sites (miR-100-PT) or (B) Luc fused to the 3'UTR of mTOR, which harbors 3 endogenous target sites for miR-100. (C) Luc expression increased upon mutation of two (MS2) sites with full expression upon mutation of all three sites (MS3). Luc-CTL contains three random scrambled target sites that do not match any known miRNA sequence. (D) Luc expression was restored in recipient cells expressing miR-100-PT upon pretreatment of donor DKO-1 cells with $100 \mathrm{nM}$ miR-100 antagomirs (Al-100) compared to pre-treatment of donor DKO-1 cells with $100 \mathrm{~nm}$ control antagomirs (AI-CTL) targeting cel-miR-67. (E) Taqman qRT-PCR for miR-100. Compared to DKs-8 recipient cells grown without donor cells, mir-100 levels increased by approximately $34 \%$ in the presence of mutant DKO-1 donor cells pre-treated with Al-CTL compared to an $8 \%$ increase in Al-100 pre-treated donor cells. $\mathrm{Y}$ axis is $\%$ increase in miR-100 $=\left(\mathrm{CP}_{\mathrm{Al}-\mathrm{CTL}} \text { or } \mathrm{CP}_{\mathrm{Al}-100}-\mathrm{CP}_{\text {no donor }} / \mathrm{CP}_{\text {no donor }}\right)^{\star 100}$, where $\mathrm{CP}=$ absolute copy number. All Luc values were normalized to co-transfected vectors expressing $\beta$-galactosidase; $n=3$ independent experiments in $\mathbf{A}-\mathbf{C}$ and $n=4$ in $\mathbf{D}$, $\mathbf{E}$. All Luc assays were performed in technical triplicate. Significance was determined by two-tailed, paired t-tests where * are $p$ values $\leq 0.05$ and $* \star \leq 0.01$. Also see Figure 6-figure supplements 1-3.

DOI: 10.7554/eLife.07197.019

Figure 6. continued on next page 
Figure 6. Continued

The following figure supplements are available for figure 6:

Figure supplement 1. miR-100 binding sites in the mTOR $3^{\prime} U T R$. DOI: 10.7554/eLife.07197.020

Figure supplement 2. Presence of mutant DKO-1 donor cells augments miR-100 levels in DKs-8 recipient cells. DOI: 10.7554/eLife.07197.021

Figure supplement 3. Transfer of extracellular miRNAs by mutant DKO-1 cells promotes target repression in wild-type DKs-8 cells. DOI: 10.7554/eLife.07197.022

KRAS status-specific patterns of secreted miRNAs support the idea of using exosomes as potential biomarkers in CRC. Our finding that miR-10b is preferentially enriched in wild-type KRAS-derived exosomes, while miR-100 is enriched in mutant KRAS-derived exosomes raises interesting questions regarding how they are selected for secretion. miR-10b and miR-100 are both part of the miR-10/100 family and differ by only one base in the seed region, allowing regulation of distinct sets of target mRNAs (Tehler et al., 2011). Whether the accumulation or export of these miRNAs is a result or a consequence of oncogenic signaling remains unknown. Preventing the export or retention of certain miRNAs, such as miR-100 and miR-10b, may serve a therapeutic role in reversing the tumorigenic effects seen with aberrant miRNA expression.

KRAS-dependent differential miRNA expression more prominently affected miRNA expression patterns observed in exosomes than in the parent cells. This could reflect a mechanism by cells to selectively export miRNAs so as to maintain specific growth or gene expression states. This is consistent with a recent report that found that the cellular levels of miR-218-5p could be maintained, despite changes in the abundance of its target, likely through a 'miRNA relocation effect' where unbound miRNAs that are in excess have the potential to be sorted to exosomes (Squadrito et al., 2014). Another mechanism may be through sequence-specific motifs that direct miRNA trafficking by interaction with specific chaperone proteins (Bolukbasi et al., 2012; Villarroya-Beltri et al., 2013). Although we did not find any globally significant motif overrepresented in exosomal miRNAs, we cannot rule out that individual miRNAs might undergo sequence-specific export. miR-320 family members all contain the GGAG motif that has been proposed to serve as an exosomal targeting signal (Villarroya-Beltri et al., 2013). We found that members of the miR-320 family are preferentially enriched in exosomes independent of KRAS status; however, the GGAG sequence was not found in other miRNAs that are targeted to exosomes. It has been reported that the biogenesis of miR-320 family members occurs by a non-canonical pathway that requires neither Drosha (Chong et al., 2010) nor XPO5 (Xie et al., 2013). Instead, the 5' ends contain a 7-methyl guanosine cap that facilitates nuclear-cytoplasmic transport through XPO1 (Xie et al., 2013). XPO1 is present in DKO-1, DKs-8, and DLD-1 exosomes as detected by mass spectrometry (Demory Beckler et al., 2013). It will be interesting to investigate whether alternate processing pathways and associated biogenic machinery contribute to the heterogeneity of EV cargo and affect miRNA secretion.

It was recently demonstrated that miRNAs in B-cell exosomes display enriched levels of nontemplate-directed 3'-uridylated miRNAs, while 3'-adenylated miRNA species are preferentially cell enriched (Koppers-Lalic et al., 2014). In certain contexts, the addition of non-templated uridine residues to cognate miRNAs accelerates miRNA turnover (Baccarini et al., 2011; Wei et al., 2012). Thus, it is possible that the stability/half-life of a miRNA affects whether it is retained or secreted. While the exact functional significance of 3 '-end modifications of miRNAs detected in both cells and exosomes remains to be determined, it could be that differential export of 'tagged' miRNAs could allow cells to export specific miRNAs. However, the lack of any apparent motif upon global analysis of miRNAs enriched in exosomes, coupled to the finding that even untagged miRNAs are differentially exported, suggests multiple strategies for loading of miRNAs into EVs, and that not all EVs and exosomes contain identical cargo. This further implies that different cell types secrete a heterogeneous population of vesicles. Although the biological relevance of these findings remains to be determined, the specific sorting of miRNAs into exosomes may enable cancer cells to discard tumorsuppressive miRNAs so as to increase their oncogenic potential or perhaps modulate gene expression in neighboring and distant cells to promote tumorigenesis. In support of this hypothesis, miR-100, which we found to be enriched in mutant KRAS exosomes, was found to down-regulate LGR5 in CRC 
cells and thereby inhibit migration and invasion of such cells (Zhou et al., 2015). In this context, removal of miR-100 from the cell would be a tumor-promoting event.

In other contexts, miR-100 can have contradictory activities, both inducing EMT by downregulating E-cadherin through targeting SMARCA5 and inhibiting tumorigenicity by targeting HOXA1 (Chen et alo, 2014). Thus, although miR-100 can function as a tumor suppressor under normal conditions, augmenting its levels, for example, by EV uptake, could potentially promote EMT. In this regard, the role of miR-100 in tumorigenesis would be twofold, where its secretion in exosomes could function to maintain low-intracellular levels within mutant cells, while inducing EMT in wild-typerecipient cells. Along these lines, miR-100 is part of the miR-125b/let-7a-2/miR-100 cluster that is transcribed and expressed coordinately (Emmrich et alo, 2014). Interestingly, in malignant colonic tissues from individuals with CRC, miR-100 levels were significantly decreased while let-7a levels were strongly upregulated (Tarasov et alo, 2014). Based on our finding that there is differential accumulation of individual miRNAs within this cluster between mutant KRAS cells and exosomes, it will be interesting to determine whether cancer cells down-regulate specific miRNAs by active secretion, while simultaneously maintaining the levels of other miRNAs transcribed within the same cluster.

miRNAs are secreted from malignant breast epithelial cells after packaging into vesicles larger than conventional exosomes that are enriched in CD44, whose expression is linked to breast cancer metastasis (Palma et al., 2012). Normal cells tend to release miRNAs in more homogenous types of exosomes, suggesting that malignant transformation may alter the formation of secreted vesicles that could alter miRNA export and lead to differences in exosome content and morphology (Palma et al., 2012; Melo et al., 2014). In support of this, it was recently shown that in exosomes from breast cancer cells, CD43 mediates the accumulation of Dicer (Melo et al., 2014). These exosomes also contain other RNA-induced silencing complex (RISC) proteins and pre-miRNAs, indicating that miRNA processing can occur in exosomes (Melo et al., 2014). These components were absent in exosomes derived from normal cells. It remains to be determined whether components of the RISC-loading complex assemble within endosomes before their secretion as exosomes or by the fusion of exosomes containing heterogeneous cargo after they are secreted. The observation that cells can selectively release miRNAs and also release a heterogeneous population of vesicles raises the possibility that differential release of miRNAs is associated with different classes of exosomes and microvesicles.

Recently, quantitative analysis of secreted miRNAs suggested that the levels of extracellular miRNAs are limited and raise the question as to how such levels can alter gene expression in recipient cells (Chevillet et al., 2014). The results of our Transwell co-culture experiments are most consistent with extracellular transfer of specific miRNAs to alter expression of reporter constructs. Nevertheless, the level of exosomal transfer that is needed to alter recipient cell gene expression in vivo remains an open question. Our finding that mutant KRAS protein can be functionally transferred in exosomes indicates that the full effect of exosomes on recipient cells can be due to a combination of both RNA delivery and protein-based signaling (Higginbotham et al., 2011). This could include activation of Toll-like receptors with possible downstream effects following nuclear factor kappa-light-chain-enhancer of activated B cells or mitogen-activated protein kinase cascades (Fabbri et al., 2012; Chen et al., 2013). The complexity of miR-100 function in the tumor microenvironment underscores this argument by its potential for inhibiting mTOR expression which is required for proliferation of Apc-deficient tumors in mouse models (Faller et al., 2015). In tumors where some cells have incurred activating mutations in KRAS, while others have not, miR-100 could accumulate in wild-type KRAS tumor cells through exosomal transfer, inhibiting mTOR and cell growth. Conversely, miR-100 could be secreted from mutant KRAS cells giving them a growth advantage. In this way, exosomal transfer of miRNAs might act to select for cells carrying specific tumor driver mutations. Our studies have direct implications for CRC and, together with other studies, indicate that delivery of exosomes to recipient cells can induce cell migration, inflammation, immune responses, angiogenesis, invasion, pre-metastatic niche formation, and metastasis (Kahlert and Kalluri, 2013; Boelens et al., 2014; Melo et al., 2014; Costa-Silva et al., 2015).

\section{Materials and methods}

\section{Exosome isolation}

Exosomes were isolated from conditioned medium of DKO-1, Dks-8, and DLD-1 cells as previously described, with slight modification (Higginbotham et al., 2011). Briefly, cells were cultured in Dulbecco's Modified Eagle's Medium (DMEM) supplemented with 10\% bovine growth serum until 
$80 \%$ confluent. The cells were then washed three times with Phosphate buffered saline (PBS) and cultured for $24 \mathrm{hr}$ in serum-free medium. The medium was collected and replaced with ionomycincontaining medium for $1 \mathrm{hr}$, after which ionomycin-containing medium was collected and pooled with the previously collected serum-free medium. Pooled media was centrifuged for $10 \mathrm{~min}$ at $300 \times \mathrm{g}$ to remove cellular debris, and the resulting supernatant was then filtered through a $0.22-\mathrm{mm}$ polyethersulfone filter (Nalgene, Rochester, NY, USA) to reduce microparticle contamination. The filtrate was concentrated $\sim 300$-fold with a 100,000 molecular weight cut-off centrifugal concentrator (Millipore, Darmstadt, Germany). The concentrate was then subjected to high-speed centrifugation at $150,000 \times g$ for $2 \mathrm{hr}$. The resulting exosome-enriched pellet was resuspended in PBS containing $25 \mathrm{mM}$ hydroxyethyl-piperazineethanesulfonic acid (HEPES) (pH 7.2) and washed by centrifuging again at $150,000 \times g$ for $3 \mathrm{hr}$. The wash steps were repeated a minimum of three times until no trace of phenol red was detected. The resulting pellet was resuspended in PBS containing $25 \mathrm{mM} \mathrm{HEPES} \mathrm{(pH} \mathrm{7.2),} \mathrm{and}$ protein concentrations were determined with a MicroBCA kit (Pierce/Thermo, Rockford, IL, USA). The number of exosomes per $\mu \mathrm{g}$ of protein was determined by means of nanoparticle tracking analysis (NanoSight, Wiltshire, UK). Analysis was performed on three independent preparations of exosomes.

\section{RNA purification}

Total RNA from exosomes and cells was isolated using TRIzol (Life Technologies/Thermo, Grand Island, NY). In the case of exosomal RNA isolation, TRlzol was incubated with $100 \mu$ l or less of concentrated exosomes for an extended $15 \mathrm{~min}$ incubation prior to chloroform extraction. RNA pellets were resuspended in $60 \mu \mathrm{l}$ of RNase-free water and were then re-purified using the miRNeasy kit (Qiagen Inc., Valencia, CA, USA). Final RNAs were eluted with two rounds of $30 \mu \mathrm{l}$ water extraction.

\section{miRNA library preparation and sequencing}

Total RNA from each sample was used for small RNA library preparation using NEBNext Small RNA Library Prep Set from Illumina (New England BioLabs Inc., Ipswich, MA, USA). Briefly, 3' adapters were ligated to total input RNA followed by hybridization of multiplex single read (SR) reverse transcription (RT) primers and ligation of multiplex 5' SR adapters. RT was performed using ProtoScript II RT for $1 \mathrm{hr}$ at $50^{\circ} \mathrm{C}$. Immediately after RT reactions, PCR amplification was performed for 15 cycles using LongAmp Taq $2 \times$ master mix. Illumina-indexed primers were added to uniquely barcode each sample. Post-PCR material was purified using QIAquick PCR purification kits (Qiagen Inc.). Post-PCR yield and concentration of the prepared libraries were assessed using Qubit 2.0 Fluorometer (Invitrogen, Carlsbad, California, CA, USA) and DNA 1000 chip on Agilent 2100 Bioanalyzer (Applied Biosystems, Carlsbad, CA, USA), respectively. Size selection of small RNA with a target size range of approximately 146-148 bp was performed using 3\% dye free agarose gel cassettes on a Pippin Prep instrument (Sage Science Inc., Beverly, MA, USA). Post-size selection yield and concentration of libraries were assessed using Qubit 2.0 Fluorometer and DNA high-sensitivity chip on an Agilent 2100 Bioanalyzer, respectively. Accurate quantification for sequencing applications was performed using qPCR-based KAPA Biosystems Library Quantification kits (Kapa Biosystems, Inc., Woburn, MA, USA). Each library was diluted to a final concentration of $1.25 \mathrm{nM}$ and pooled in equimolar ratios prior to clustering. Cluster generation was carried out on a cBot v8.0 using Illumina's Truseq Single Read Cluster Kit v3.0. Singleend sequencing was performed to generate at least 15 million reads per sample on an Illumina HiSeq2000 using a 50-cycleTruSeq SBSHSv3 reagent kit. Clustered flow cells were sequenced for 56 cycles, consisting of a 50-cycle read, followed by a 6-cycle index read. Image analysis and base calling were performed using the standard Illumina pipeline consisting of Real Time Analysis version v1.17 and demultiplexed using bcl2fastq converter with default settings.

\section{Mapping of RNA reads}

Read sequence quality checks were performed by FastQC (Babraham Bioinformatics [http://www. bioinformatics.babraham.ac.uk/projects/fastqc/]). Adapters from the $3^{\prime}$ ends of reads were trimmed using Cutadpt with a maximum allowed error rate of 0.1 (Martin, 2011). Reads shorter than 15 nucleotides in length were excluded from further analysis. Reads were mapped to the human genome hg19 using Bowtie version 1.1.1 (Langmead and Salzberg, 2012). Mapped reads were annotated using ncPRO-seq (Chen et al., 2012) based on miRbase (Griffiths-Jones et al., 2008), Rfam (Gardner et al., 2011; Burge et al., 2013), and RepeatMasker (http://www.repeatmasker.org/), and expression 
levels were quantified based on read counts. Mature miRNA annotation was extended 2 bp in both upstream and downstream regions to accommodate inaccurate processing of precursor miRNAs. Reads with multiple mapping locations were weighted by the number of mapping locations.

\section{PC analysis}

DESeq Version 1.16.0 was used to perform PC analyses (Anders and Huber, 2010).

\section{Enrichment analysis}

Differential expression was analyzed using DESeq Version 1.16.0 (Anders and Huber, 2010). Negative binomial distribution was used to compare miRNA abundance between cells vs exosomes and wild-type vs mutant KRAS status. The trimmed mean of $\mathrm{M}$ values method was used for normalization (Robinson and Oshlack, 2010). Differential expression was determined based on log2 fold change (log2 fold change) and false discovery rate (FDR) with llog2 fold changel $\geq 1$ and FDR $\leq 0.001$.

\section{Trimming and tailing}

Trimming and tailing analysis was based on miRBase annotation (Griffiths-Jones et al., 2006, 2008; Griffiths-Jones, 2010). Only high-confidence miRNAs (544) and corresponding hairpin sequences were used. Bowtie version 1.1.1 with 0 mismatch was used for mapping. miRNA reads were first mapped to hairpin sequences with unmapped reads, then mapped to the human genome hg19. Remaining reads were trimmed $1 \mathrm{bp}$ from the $3^{\prime}$ end and remapped to hairpin sequences. The remapping process was repeated 10 times. Finally, all mapped reads were collected for further analysis.

\section{qRT/PCR}

Taqman small RNA assays (Life Technologies) (individual assay numbers are listed below) were performed for indicated miRNAs on cellular and exosomal RNA samples. Briefly, $10 \mathrm{ng}$ of total RNA was used per individual RT reactions; $0.67 \mu \mathrm{l}$ of the resultant CDNA was used in $10 \mu \mathrm{l}$ qPCR reactions. qPCR reactions were conducted in 96-well plates on a Bio-Rad CFX96 instrument. All C $(t)$ values were $\leq 30$. Triplicate $C(t)$ values were averaged and normalized to U6 snRNA. Fold changes were calculated using the $\triangle \Delta C(t)$

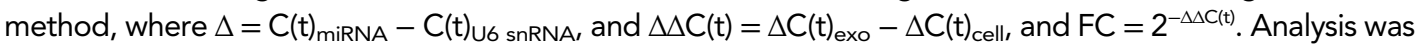
performed on three independent cell and exosomal RNA samples. Taqman probe \#: U6 snRNA: 001973; hsa-let-7a-5p: 000377; hsa-miR-100-5p: 000437; hsa-miR-320b: 002844; hsa-miR-320a: 002277.

\section{Generation of miRNA standard curves}

RNase-free, HPLC-purified 5'-phosphorylated miRNA oligoribonucleotides were synthesized (Integrated DNA Technologies) for human miR-100-5p (5'-phospho-AACCCGUAGAUCCGAACUUGUG-OH-3') and cel-miR-39-3p (5'-phospho-UCACCGGGUGUAAAUCAGCUUG-OH-3'). Stock solutions of $10 \mu \mathrm{M}$ synthetic oligonucleotide in RNase-free and DNase-free water were prepared according to the concentrations and sample purity quoted by the manufacturer (based on spectrophotometry analysis). Nine twofold dilution series beginning with 50 pM synthetic oligonucleotide were used in $10 \mu \mathrm{RT}$ reactions (Taqman small RNA assays), and qPCR was performed. Each dilution was performed in triplicate from three independent experiments. Linear regression was used to determine mean $\mathrm{C}(\mathrm{t})$ values plotted against $\log (\operatorname{miRNA}$ copies/ $\mu$ )).

\section{miRNA in situ hybridizations and ceramide dependence}

Cells were plated in 6-well plates containing coverslips at a density of $\sim 2.5 \times 10^{5}$ cells and cultured in DMEM supplemented with $10 \%$ bovine growth serum for $24 \mathrm{hr}$. The cells were then washed three times with PBS and cultured for $24 \mathrm{hr}$ in serum-free medium containing either $5 \mu \mathrm{M}$ GW4869 (Cayman Chemicals \# 13127, Ann Arbor, MI, USA) or DMSO. Medium was removed and cells were washed three times with PBS and fixed with 4\% Paraformaldehyde (PFA) for $15 \mathrm{~min}$ at room temperature. After, cells were washed three times in DEPC-treated PBS and permeabilized in $70 \%$ ethanol for $\sim 4 \mathrm{hr}$ at $4^{\circ} \mathrm{C}$, and rehydrated in DEPC-treated PBS for 5 min. Pre-hybridization was performed in hybridization buffer (25\% formamide, 0.05 M EDTA, 4× saline-sodium citrate (SSC), $10 \%$ dextran sulfate, $1 X$ Denhardt's solution $1 \mathrm{mg} / \mathrm{ml}$ Escherichia coli tRNA) in a humidified chamber at $60^{\circ} \mathrm{C}$ for 60 min. Hybridization buffer was removed and replaced with $10 \mathrm{nM}$ of probe (probe numbers are listed below) diluted in hybridization buffer and incubated at either $55^{\circ} \mathrm{C}$ (miR-100 and miR-10b) or $57^{\circ} \mathrm{C}$ for 
scrambled and U6 probes for $2 \mathrm{hr}$. Coverslips were then washed in series with pre-heated SSC at $37^{\circ} \mathrm{C}$ as follows: $4 \times$ SSC briefly, $2 \times$ SSC for $30 \mathrm{~min}, 1 \times$ SSC for $30 \mathrm{~min}$, and $0.1 \times$ SSC for $20 \mathrm{~min}$. miRNA detection was conducted using Tyramide Signal Amplification (Perkin Elmer, \# NEL741001KT, Waltham, MA, USA). Briefly, coverslips were blocked in blocking buffer $(0.1 \mathrm{M} \mathrm{TRIS-HCl}, \mathrm{pH} 7.5,0.15$ $\mathrm{M} \mathrm{NaCl}$, 0.5\% Blocking Reagent [Roche, \#11096176001, Basel, Switzerland]) at $4^{\circ} \mathrm{C}$ overnight. Blocking buffer was replaced with anti-DIG-POD (Roche, \# 11207733910) diluted 1:100 in blocking buffer and incubated for $60 \mathrm{~min}$. Coverslips were washed three times, 5 min per wash, in wash buffer (0.1 M Tris- $\mathrm{HCl}, \mathrm{pH} 7.5,0.15 \mathrm{M} \mathrm{NaCl}, 0.5 \%$ Saponin) followed by incubation with 1× Fluorescein diluted in $1 \times$ amplification reagent for $5 \mathrm{~min}$. Fluorescent coverslips were then washed two times, 5 min per wash, in wash buffer. To preserve fluorescent signals, coverslips were fixed with $2 \%$ PFA containing $2 \%$ Bovine serum albumin in $1 \times$ PBS for 15 min. After fixation, coverslips were washed 2 times, 5 min per wash, in wash buffer, followed by a final wash in $1 \times$ PBS for 5 min. Coverslips were then mounted in Prolong Gold (Life Technologies) and visualized on a Zeiss LSM510 at $63 \times$ objective. 3'DIG labeled probes for in situ hybridizations-U6 snRNA: 99002-05; Scramble: 99004-05; miR-10b-5p: 38486-05; miR-100-5p: 18009-05 (Exiqon, Woburn, MA, USA).

\section{Co-culture and Luc reporter assays}

Recipient cells were plated in six-well plates at a density of $\sim 2.5 \times 10^{5}$ cells and cultured in DMEM supplemented with $10 \%$ bovine growth serum for $24 \mathrm{hr}$. Media was replaced and cells were co-transfected (Promega, E2311, Madison, WI, USA) with $1.5 \mu \mathrm{g}$ of Luc-reporter plasmid and $1.5 \mu \mathrm{g}$ $\beta$-gal plasmid DNA/well. Donor cells were plated in $0.4-\mu \mathrm{m}$ polyester membrane Transwell filters (Corning, 3450, Corning, NY, USA) at $\sim 2.5 \times 10^{5}$ cells/well for $24 \mathrm{hr}$. Media from donor Transwells and recipient 6-well plates were removed and replaced with DMEM without FBS. Co-culture of donor and recipient cells was conducted for either 24 or $48 \mathrm{hr}$ before recipient cells were harvested. Lysates were prepared in 1× Reporter lysis buffer (Promega, E2510), and Luc assays were performed according to the manufacturer's protocol (Promega, E2510). $\beta$-gal expression was simultaneously determined from the lysates according to the manufacturer's protocol (Promega, E2000). Differences in transfection efficiency were accounted for by normalizing Luc expression to $\beta$-Gal expression (Luc/ $\beta$-Gal). All assays were performed on three biological replicates, each with three technical replicates.

\section{Antagomir treatment}

Donor cells were plated in 0.4- $\mu \mathrm{m}$ polyester membrane Transwell filters (Corning, 3450, Corning, NY, USA) at $\sim 1.4 \times 10^{4}$ cells/well for $24 \mathrm{hr}$. Medium was replaced and donor cells were transfected with either miR-100 hairpin antagomirs (\# IH-300517-05, GE Life Sciences) or negative control hairpin antagomirs corresponding to cel-miR-67 (\# IN-001005-01, GE Life Sciences) to produce a final concentration of $100 \mathrm{nM}$ of antagomir for $24 \mathrm{hr}$. Medium from donor Transwells and recipient 6-well plates was removed and replaced with DMEM without FBS. Co-culture of donor and recipient cells was conducted for $24 \mathrm{hr}$ before recipient cells were harvested for RNA isolation.

\section{Plasmid construction}

For the pLuc-mTOR construct, the 3'UTR of mTOR was PCR amplified (primer sequences in Supplementary file 3) from genomic DNA isolated from DKs-8 cells. The amplicon was cloned into pMiR-Report (Life Technologies) via Spel/HinDIII restriction sites. Mutation of miR-100 binding sites in mTOR 3'UTR (MS) was performed on pLuc-mTOR using forward or reverse primers targeting either all three MRE's, or MRE 2 and 3 with QuikChange Lightning Multi-Site Directed Mutagenesis (Agilent, Santa Clara, CA, USA) according to manufacturer's protocol. To create the reporter construct containing three miR-100 perfect sites (miR-100-PT), oligonucleotides (Supplementary file 3) were annealed to produce a synthetic fragment containing the perfect sites with CTAGT and AGCTT overhangs. The fragment was cloned into pMiR-report via Spel/HinDIII restriction sites. All plasmids were sequence verified (GeneWiz, South Plainfield, NJ, USA).

\section{Acknowledgements}

This work was supported by grants from the National Institutes of Health, U19CA179514, RO1 CA163563 and a GI Special Program of Research Excellence (SPORE) P50 95103 to RJC, and a pilot in P30 DK058404 to JLF. Vanderbilt Digestive Disease Research Center (P30 DK058404) and associated Cores. 


\section{Additional information}

Funding

\begin{tabular}{|c|c|c|}
\hline Funder & Grant reference & Author \\
\hline $\begin{array}{l}\text { National Institutes of } \\
\text { Health }(\mathrm{NIH})\end{array}$ & U19CA179514 & $\begin{array}{l}\text { Robert J Coffey, James G } \\
\text { Patton }\end{array}$ \\
\hline National Cancer Institute (NCl) & P50 95103 & Robert J Coffey \\
\hline $\begin{array}{l}\text { National Institutes of } \\
\text { Health (NIH) }\end{array}$ & RO1 CA163563 & Robert J Coffey \\
\hline $\begin{array}{l}\text { National Institutes of } \\
\text { Health }(\mathrm{NIH})\end{array}$ & P30 DK058404 & Jeffrey L Franklin \\
\hline
\end{tabular}

The funders had no role in study design, data collection and interpretation, or the decision to submit the work for publication.

Author contributions

DJC, JLF, Conception and design, Acquisition of data, Analysis and interpretation of data, Drafting or revising the article; YD, $\mathrm{QL}, \mathrm{JNH}, \mathrm{MDB}, \mathrm{NP}, \mathrm{SL}$, Acquisition of data, Analysis and interpretation of data; AMW, KV, RJC, JGP, Conception and design, Drafting or revising the article; BZ, Conception and design, Analysis and interpretation of data, Drafting or revising the article

\section{Additional files}

Supplementary files

- Supplementary file 1. Read counts for (A) individual miRNA species and (B) repeat families.

DOI: 10.7554/eLife.07197.023

- Supplementary file 2. Abundant miRNAs. Normalized read counts (see 'Materials and methods') were used to determine the miRNAs with the highest number of reads (top 50) from cell and exosomal data sets. The top 50 most abundant miRNAs were compared between exosomes and cells for each cell line. Most abundant miRNAs in exosomes (blue), cells (orange), or both.

DOI: 10.7554/eLife.07197.024

- Supplementary file 3. Related to experimental procedures. Primers used for plasmid construction. DOI: 10.7554/eLife.07197.025

\section{References}

Alvarez-Garcia I, Miska EA. 2005. MicroRNA functions in animal development and human disease. Development 132:4653-4662. doi: 10.1242/dev.02073.

Anders S, Huber W. 2010. Differential expression analysis for sequence count data. Genome Biology 11:R106. doi: 10.1186/gb-2010-11-10-r106.

Arroyo JD, Chevillet JR, Kroh EM, Ruf IK, Pritchard CC, Gibson DF, Mitchell PS, Bennett CF, PogosovaAgadjanyan EL, Stirewalt DL, Tait JF, Tewari M. 2011. Argonaute2 complexes carry a population of circulating microRNAs independent of vesicles in human plasma. Proceedings of the National Academy of Sciences of USA 108:5003-5008. doi: 10.1073/pnas.1019055108.

Baccarini A, Chauhan H, Gardner TJ, Jayaprakash AD, Sachidanandam R, Brown BD. 2011. Kinetic analysis reveals the fate of a microRNA following target regulation in mammalian cells. Current Biology 21:369-376. doi: 10. 1016/j.cub.2011.01.067.

Bartel DP, Chen CZ. 2004. Micromanagers of gene expression: the potentially widespread influence of metazoan microRNAs. Nature Reviews. Genetics 5:396-400. doi: 10.1038/nrg1328.

Batagov AO, Kuznetsov VA, Kurochkin IV. 2011. Identification of nucleotide patterns enriched in secreted RNAs as putative cis-acting elements targeting them to exosome nano-vesicles. BMC Genomics 12(Suppl 3):S18. doi: 10. 1186/1471-2164-12-S3-S18.

Boelens MC, Wu TJ, Nabet BY, Xu B, Qiu Y, Yoon T, Azzam DJ, Twyman-Saint Victor C, Wiemann BZ, Ishwaran H, Ter Brugge PJ, Jonkers J, Slingerland J, Minn AJ. 2014. Exosome transfer from stromal to breast cancer cells regulates therapy resistance pathways. Cell 159:499-513. doi: 10.1016/j.cell.2014.09.051.

Bolukbasi MF, Mizrak A, Ozdener GB, Madlener S, Ströbel T, Erkan EP, Fan J-B, Breakefield XO, Saydam O. 2012. miR-1289 and "Zipcode"-like sequence enrich mRNAs in microvesicles. Molecular Therapy. Nucleic Acids 1:e10. doi: 10.1038/mtna.2011.2. 
Burge SW, Daub J, Eberhardt R, Tate J, Barquist L, Nawrocki EP, Eddy SR, Gardner PP, Bateman A. 2013. Rfam 11.0: 10 years of RNA families. Nucleic Acids Research 41:D226-D232. doi: 10.1093/nar/gks1005.

Burroughs AM, Ando Y, de Hoon MJ, Tomaru Y, Nishibu T, Ukekawa R, Funakoshi T, Kurokawa T, Suzuki H, Hayashizaki Y, Daub CO. 2010. A comprehensive survey of 3' animal miRNA modification events and a possible role for $3^{\prime}$ adenylation in modulating miRNA targeting effectiveness. Genome Research 20:1398-1410. doi: 10. 1101/gr.106054.110.

Chen CJ, Servant N, Toedling J, Sarazin A, Marchais A, Duvernois-Berthet E, Cognat V, Colot V, Voinnet O, Heard E, Ciaudo C, Barillot E. 2012. ncPRO-seq: a tool for annotation and profiling of ncRNAs in sRNA-seq data. Bioinformatics 28:3147-3149. doi: 10.1093/bioinformatics/bts587.

Chen X, Liang H, Zhang J, Zen K, Zhang CY. 2013. microRNAs are ligands of Toll-like receptors. RNA 19:737-739. doi: 10.1261/rna.036319.112.

Chen D, Sun Y, Yuan Y, Han Z, Zhang P, Zhang J, You MJ, Teruya-Feldstein J, Wang M, Gupta S, Hung MC, Liang H, Ma L. 2014. miR-100 induces epithelial-mesenchymal transition but suppresses tumorigenesis, migration and invasion. PLOS Genetics 10:e1004177. doi: 10.1371/journal.pgen.1004177.

Chevillet JR, Kang Q, Ruf IK, Briggs HA, Vojtech LN, Hughes SM, Cheng HH, Arroyo JD, Meredith EK, Gallichotte EN, Pogosova-Agadjanyan EL, Morrissey C, Stirewalt DL, Hladik F, Yu EY, Higano CS, Tewari M. 2014. Quantitative and stoichiometric analysis of the microRNA content of exosomes. Proceedings of the National Academy of Sciences of USA 111:14888-14893. doi: 10.1073/pnas.1408301111.

Chong MM, Zhang G, Cheloufi S, Neubert TA, Hannon GJ, Littman DR. 2010. Canonical and alternate functions of the microRNA biogenesis machinery. Genes \& Development 24:1951-1960. doi: 10.1101/gad.1953310.

Costa-Silva B, Aiello NM, Ocean AJ, Singh S, Zhang H, Thakur BK, Becker A, Hoshino A, Mark MT, Molina H, Xiang J, Zhang T, Theilen TM, García-Santos G, Williams C, Ararso Y, Huang Y, Rodrigues G, Shen TL, Labori KJ, Lothe IM, Kure EH, Hernandez J, Doussot A, Ebbesen SH, Grandgenett PM, Hollingsworth MA, Jain M, Mallya K, Batra SK, Jarnagin WR, Schwartz RE, Matei I, Peinado H, Stanger BZ, Bromberg J, Lyden D. 2015. Pancreatic cancer exosomes initiate pre-metastatic niche formation in the liver. Nature Cell Biology 17:816-826. doi: 10.1038/ ncb3169.

Crescitelli R, Lasser C, Szabo TG, Kittel A, Eldh M, Dianzani I, Buzas El, Lotvall J. 2013. Distinct RNA profiles in subpopulations of extracellular vesicles: apoptotic bodies, microvesicles and exosomes. Journal of Extracellular Vesicles 2. doi: 10.3402/jev.v2i0.20677.

Demory Beckler M, Higginbotham JN, Franklin JL, Ham AJ, Halvey PJ, Imasuen IE, Whitwell C, Li M, Liebler DC, Coffey RJ. 2013. Proteomic analysis of exosomes from mutant KRAS colon cancer cells identifies intercellular transfer of mutant KRAS. Molecular \& Cellular Proteomics 12:343-355. doi: 10.1074/mcp.M112.022806.

Ekstrom K, Valadi H, Sjostrand M, Malmhall C, Bossios A, Eldh M, Lotvall J. 2012. Characterization of mRNA and microRNA in human mast cell-derived exosomes and their transfer to other mast cells and blood CD34 progenitor cells. Journal of Extracellular Vesicles 1:18389. doi: 10.3402/jev.v1i0.18389.

Emmrich S, Rasche M, Schoning J, Reimer C, Keihani S, Maroz A, Xie Y, Li Z, Schambach A, Reinhardt D, Klusmann JH. 2014. miR-99a/100 125b tricistrons regulate hematopoietic stem and progenitor cell homeostasis by shifting the balance between TGFbeta and Wnt signaling. Genes \& Development 28:858-874. doi: 10.1101/gad. 233791.113.

Fabbri M, Paone A, Calore F, Galli R, Gaudio E, Santhanam R, Lovat F, Fadda P, Mao C, Nuovo GJ, Zanesi N, Crawford M, Ozer GH, Wernicke D, Alder H, Caligiuri MA, Nana-Sinkam P, Perrotti D, Croce CM. 2012. MicroRNAs bind to Toll-like receptors to induce prometastatic inflammatory response. Proceedings of the National Academy of Sciences of USA 109:E2110-E2116. doi: 10.1073/pnas.1209414109.

Faller WJ, Jackson TJ, Knight JR, Ridgway RA, Jamieson T, Karim SA, Jones C, Radulescu S, Huels DJ, Myant KB, Dudek KM, Casey HA, Scopelliti A, Cordero JB, Vidal M, Pende M, Ryazanov AG, Sonenberg N, Meyuhas O, Hall MN, Bushell M, Willis AE, Sansom OJ. 2015. mTORC1-mediated translational elongation limits intestinal tumour initiation and growth. Nature 517:497-500. doi: 10.1038/nature13896.

Gardner PP, Daub J, Tate J, Moore BL, Osuch IH, Griffiths-Jones S, Finn RD, Nawrocki EP, Kolbe DL, Eddy SR, Bateman A. 2011. Rfam: wikipedia, clans and the "decimal" release. Nucleic Acids Research 39:D141-D145. doi: 10.1093/nar/gkq1129.

Ge YY, Shi Q, Zheng ZY, Gong J, Zeng C, Yang J, Zhuang SM. 2014. MicroRNA-100 promotes the autophagy of hepatocellular carcinoma cells by inhibiting the expression of mTOR and IGF-1R. Oncotarget 5:6218-6228.

Gebeshuber CA, Martinez J. 2013. miR-100 suppresses IGF2 and inhibits breast tumorigenesis by interfering with proliferation and survival signaling. Oncogene 32:3306-3310. doi: 10.1038/onc.2012.372.

Griffiths-Jones S, Grocock RJ, van Dongen S, Bateman A, Enright AJ. 2006. miRBase: microRNA sequences, targets and gene nomenclature. Nucleic Acids Research 34:D140-D144. doi: 10.1093/nar/gkj112.

Griffiths-Jones S, Saini HK, van Dongen S, Enright AJ. 2008. miRBase: tools for microRNA genomics. Nucleic Acids Research 36:D154-D158. doi: 10.1093/nar/gkm952.

Griffiths-Jones S. 2010. miRBase: microRNA sequences and annotation. In: Andreas D Baxevanis, William R Pearson, Lincoln D Stein, Gary D Stormo, John R Yates III, editors. Current protocols in bioinformatics/editoral board. Chapter 12Unit 12.

Grundmann S, Hans FP, Kinniry S, Heinke J, Helbing T, Bluhm F, Sluijter JP, Hoefer I, Pasterkamp G, Bode C, Moser M. 2011. MicroRNA-100 regulates neovascularization by suppression of mammalian target of rapamycin in endothelial and vascular smooth muscle cells. Circulation 123:999-1009. doi: 10.1161/CIRCULATIONAHA.110.000323.

Higginbotham JN, Demory Beckler M, Gephart JD, Franklin JL, Bogatcheva G, Kremers GJ, Piston DW, Ayers GD, McConnell RE, Tyska MJ, Coffey RJ. 2011. Amphiregulin exosomes increase cancer cell invasion. Current Biology 21:779-786. doi: 10.1016/j.cub.2011.03.043. 
Hoshino D, Kirkbride KC, Costello K, Clark ES, Sinha S, Grega-Larson N, Tyska MJ, Weaver AM. 2013. Exosome secretion is enhanced by invadopodia and drives invasive behavior. Cell Reports 5:1159-1168. doi: 10.1016/j. celrep.2013.10.050.

Hsieh IS, Chang KC, Tsai YT, Ke JY, Lu PJ, Lee KH, Yeh SD, Hong TM, Chen YL. 2013. MicroRNA-320 suppresses the stem cell-like characteristics of prostate cancer cells by downregulating the Wnt/beta-catenin signaling pathway. Carcinogenesis 34:530-538. doi: 10.1093/carcin/bgs371.

Ji H, Chen M, Greening DW, He W, Rai A, Zhang W, Simpson RJ. 2014. Deep sequencing of RNA from three different extracellular vesicle (EV) subtypes released from the human LIM1863 colon cancer cell line uncovers distinct miRNA-enrichment signatures. PLOS ONE 9:e110314. doi: 10.1371/journal.pone.0110314.

Kahlert C, Kalluri R. 2013. Exosomes in tumor microenvironment influence cancer progression and metastasis. Journal of Molecular Medicine 91:431-437. doi: 10.1007/s00109-013-1020-6.

Koppers-Lalic D, Hackenberg M, Bijnsdorp IV, van Eijndhoven MA, Sadek P, Sie D, Zini N, Middeldorp JM, Ylstra B, de Menezes RX, Würdinger T, Meijer GA, Pegtel DM. 2014. Nontemplated nucleotide additions distinguish the small RNA composition in cells from exosomes. Cell Reports 8:1649-1658. doi: 10.1016/j.celrep.2014.08.027.

Kosaka N, Iguchi H, Yoshioka Y, Takeshita F, Matsuki Y, Ochiya T. 2010. Secretory mechanisms and intercellular transfer of microRNAs in living cells. The Journal of Biological Chemistry 285:17442-17452. doi: 10.1074/jbc. M110.107821.

Krol J, Loedige I, Filipowicz W. 2010. The widespread regulation of microRNA biogenesis, function and decay. Nature Reviews. Genetics 11:597-610. doi: 10.1038/nrg2843.

LaConti JJ, Shivapurkar N, Preet A, Deslattes Mays A, Peran I, Kim SE, Marshall JL, Riegel AT, Wellstein A. 2011. Tissue and serum microRNAs in the Kras(G12D) transgenic animal model and in patients with pancreatic cancer. PLOS ONE 6:e20687. doi: 10.1371/journal.pone.0020687.

Langmead B, Salzberg SL. 2012. Fast gapped-read alignment with Bowtie 2. Nature Methods 9:357-359. doi: 10. 1038/nmeth.1923.

Lu J, Getz G, Miska EA, Alvarez-Saavedra E, Lamb J, Peck D, Sweet-Cordero A, Ebert BL, Mak RH, Ferrando AA, Downing JR, Jacks T, Horvitz HR, Golub TR. 2005. MicroRNA expression profiles classify human cancers. Nature 435:834-838. doi: 10.1038/nature03702.

Luga V, Zhang L, Viloria-Petit AM, Ogunjimi AA, Inanlou MR, Chiu E, Buchanan M, Hosein AN, Basik M, Wrana JL. 2012. Exosomes mediate stromal mobilization of autocrine Wnt-PCP signaling in breast cancer cell migration. Cell 151:1542-1556. doi: 10.1016/j.cell.2012.11.024.

Ma L. 2010. Role of miR-10b in breast cancer metastasis. Breast Cancer Research 12:210. doi: 10.1186/bcr2720.

Martin M. 2011. Cutadapt removes adapator sequences from high-throughput sequencing reads. EMBnet.Journal 17:10-12. doi: 10.14806/ej.17.1.200.

Melo SA, Sugimoto H, O'Connell JT, Kato N, Villanueva A, Vidal A, Qiu L, Vitkin E, Perelman LT, Melo CA, Lucci A, Ivan C, Calin GA, Kalluri R. 2014. Cancer exosomes perform cell-independent MicroRNA biogenesis and promote tumorigenesis. Cancer Cell 26:707-721. doi: 10.1016/j.ccell.2014.09.005.

Mittelbrunn M, Gutierrez-Vazquez C, Villarroya-Beltri C, Gonzalez S, Sanchez-Cabo F, Gonzalez MA, Bernad A, Sanchez-Madrid F. 2011. Unidirectional transfer of microRNA-loaded exosomes from T cells to antigenpresenting cells. Nature Communications 2:282. doi: 10.1038/ncomms1285.

Montecalvo A, Larregina AT, Shufesky WJ, Stolz DB, Sullivan ML, Karlsson JM, Baty CJ, Gibson GA, Erdos G, Wang Z, Milosevic J, Tkacheva OA, Divito SJ, Jordan R, Lyons-Weiler J, Watkins SC, Morelli AE. 2012. Mechanism of transfer of functional microRNAs between mouse dendritic cells via exosomes. Blood 119: 756-766. doi: 10.1182/blood-2011-02-338004.

Nagaraja AK, Creighton CJ, Yu Z, Zhu H, Gunaratne PH, Reid JG, Olokpa E, Itamochi H, Ueno NT, Hawkins SM, Anderson ML, Matzuk MM. 2010. A link between mir-100 and FRAP1/mTOR in clear cell ovarian cancer. Molecular Endocrinology 24:447-463. doi: 10.1210/me.2009-0295.

Neilsen CT, Goodall GJ, Bracken CP. 2012. IsomiRs-the overlooked repertoire in the dynamic microRNAome. Trends in Genetics 28:544-549. doi: 10.1016/j.tig.2012.07.005.

Newman MA, Mani V, Hammond SM. 2011. Deep sequencing of microRNA precursors reveals extensive $3^{\prime}$ end modification. RNA 17:1795-1803. doi: 10.1261/rna.2713611.

Palma J, Yaddanapudi SC, Pigati L, Havens MA, Jeong S, Weiner GA, Weimer KM, Stern B, Hastings ML, Duelli DM. 2012. MicroRNAs are exported from malignant cells in customized particles. Nucleic Acids Research 40: 9125-9138. doi: 10.1093/nar/gks656.

Petrelli A, Perra A, Schernhuber K, Cargnelutti M, Salvi A, Migliore C, Ghiso E, Benetti A, Barlati S, LeddaColumbano GM, Portolani N, De Petro G, Columbano A, Giordano S. 2012. Sequential analysis of multistage hepatocarcinogenesis reveals that miR-100 and PLK1 dysregulation is an early event maintained along tumor progression. Oncogene 31:4517-4526. doi: 10.1038/onc.2011.631.

Robinson MD, Oshlack A. 2010. A scaling normalization method for differential expression analysis of RNA-seq data. Genome Biology 11:R25. doi: 10.1186/gb-2010-11-3-r25.

Schepeler T, Reinert JT, Ostenfeld MS, Christensen LL, Silahtaroglu AN, Dyrskjot L, Wiuf C, Sorensen FJ, Kruhoffer M, Laurberg S, Kauppinen S, Ørntoft TF, Andersen CL. 2008. Diagnostic and prognostic microRNAs in stage II colon cancer. Cancer Research 68:6416-6424. doi: 10.1158/0008-5472.CAN-07-6110.

Shirasawa S, Furuse M, Yokoyama N, Sasazuki T. 1993. Altered growth of human colon cancer cell lines disrupted at activated Ki-ras. Science 260:85-88. doi: 10.1126/science.8465203.

Skog J, Wurdinger T, van Rijn S, Meijer DH, Gainche L, Sena-Esteves M, Curry WT Jr, Carter BS, Krichevsky AM, Breakefield XO. 2008. Glioblastoma microvesicles transport RNA and proteins that promote tumour growth and provide diagnostic biomarkers. Nature Cell Biology 10:1470-1476. doi: 10.1038/ncb1800. 
Squadrito ML, Baer C, Burdet F, Maderna C, Gilfillan GD, Lyle R, Ibberson M, De Palma M. 2014. Endogenous RNAs modulate microRNA sorting to exosomes and transfer to acceptor cells. Cell Reports 8:1432-1446. doi: 10. 1016/j.celrep.2014.07.035.

Tarasov VA, Matishov DG, Shin EF, Boyko NV, Timoshkina NN, Makhotkin MA, Lomonosov AM, Kirpiy AA, Kit OI, Maximov AY. 2014. Coordinated aberrant expression of miRNAs in colon cancer. Genetika 50:1090-1101. doi: 10.1134/S1022795414080109.

Tehler D, Hoyland-Kroghsbo NM, Lund AH. 2011. The miR-10 microRNA precursor family. RNA Biology 8:728-734. doi: 10.4161/rna.8.5.16324.

Thomson JM, Newman M, Parker JS, Morin-Kensicki EM, Wright T, Hammond SM. 2006. Extensive posttranscriptional regulation of microRNAs and its implications for cancer. Genes \& Development 20:2202-2207. doi: $10.1101 / \mathrm{gad} .1444406$.

Valadi H, Ekstrom K, Bossios A, Sjostrand M, Lee JJ, Lotvall JO. 2007. Exosome-mediated transfer of mRNAs and microRNAs is a novel mechanism of genetic exchange between cells. Nature Cell Biology 9:654-659. doi: 10. 1038/ncb1596.

Vickers KC, Palmisano BT, Shoucri BM, Shamburek RD, Remaley AT. 2011. MicroRNAs are transported in plasma and delivered to recipient cells by high-density lipoproteins. Nature Cell Biology 13:423-433. doi: 10.1038/ ncb2210.

Villarroya-Beltri C, Gutierrez-Vazquez C, Sanchez-Cabo F, Perez-Hernandez D, Vazquez J, Martin-Cofreces N, Martinez-Herrera DJ, Pascual-Montano A, Mittelbrunn M, Sanchez-Madrid F. 2013. Sumoylated hnRNPA2B1 controls the sorting of miRNAs into exosomes through binding to specific motifs. Nature Communications 4: 2980. doi: 10.1038/ncomms3980.

Volinia S, Calin GA, Liu CG, Ambs S, Cimmino A, Petrocca F, Visone R, lorio M, Roldo C, Ferracin M, Prueitt RL, Yanaihara N, Lanza G, Scarpa A, Vecchione A, Negrini M, Harris CC, Croce CM. 2006. A microRNA expression signature of human solid tumors defines cancer gene targets. Proceedings of the National Academy of Sciences of USA 103:2257-2261. doi: 10.1073/pnas.0510565103.

Wang M, Ren D, Guo W, Wang Z, Huang S, Du H, Song L, Peng X. 2014. Loss of miR-100 enhances migration, invasion, epithelial-mesenchymal transition and stemness properties in prostate cancer cells through targeting Argonaute 2. International Journal of Oncology 45:362-372. doi: 10.3892/ijo.2014.2413.

Wei C, Salichos L, Wittgrove CM, Rokas A, Patton JG. 2012. Transcriptome-wide analysis of small RNA expression in early zebrafish development. RNA 18:915-929. doi: 10.1261/rna.029090.111.

Wienholds E, Kloosterman WP, Miska E, Alvarez-Saavedra E, Berezikov E, de Bruijn E, Horvitz HR, Kauppinen S, Plasterk RHA. 2005. MicroRNA expression in zebrafish embryonic development. Science 309:310-311. doi: 10. 1126/science.1114519.

Wong R, Cunningham D. 2008. Using predictive biomarkers to select patients with advanced colorectal cancer for treatment with epidermal growth factor receptor antibodies. Journal of Clinical Oncology 26:5668-5670. doi: 10. 1200/JCO.2008.19.5024.

Xie M, Li M, Vilborg A, Lee N, Shu MD, Yartseva V, Sestan N, Steitz JA. 2013. Mammalian 5'-capped microRNA precursors that generate a single microRNA. Cell 155:1568-1580. doi: 10.1016/j.cell.2013.11.027.

Zhou MK, Liu XJ, Zhao ZG, Cheng YM. 2015. MicroRNA-100 functions as a tumor suppressor by inhibiting Lgr5 expression in colon cancer cells. Molecular Medicine Reports 11:2947-2952. doi: 10.3892/mmr.2014.3052. 